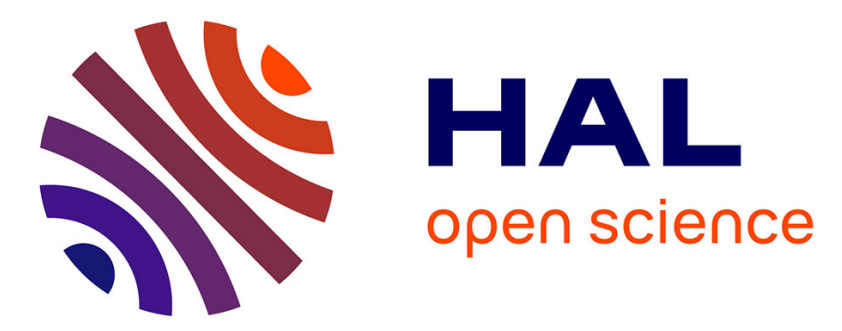

\title{
Approximation by multipoles of the multiple acoustic scattering by small obstacles and application to the Foldy theory of isotropic scattering.
}

\author{
Abderrahmane Bendali, Pierre-Henri Cocquet, Sébastien Tordeux
}

\section{To cite this version:}

Abderrahmane Bendali, Pierre-Henri Cocquet, Sébastien Tordeux. Approximation by multipoles of the multiple acoustic scattering by small obstacles and application to the Foldy theory of isotropic scattering.. Archive for Rational Mechanics and Analysis, 2016, 219 (3), pp.1017-1059. 10.1007/s00205015-0915-5 . hal-01025436

\section{HAL Id: hal-01025436 \\ https://hal.science/hal-01025436}

Submitted on 18 Jul 2014

HAL is a multi-disciplinary open access archive for the deposit and dissemination of scientific research documents, whether they are published or not. The documents may come from teaching and research institutions in France or abroad, or from public or private research centers.
L'archive ouverte pluridisciplinaire HAL, est destinée au dépôt et à la diffusion de documents scientifiques de niveau recherche, publiés ou non, émanant des établissements d'enseignement et de recherche français ou étrangers, des laboratoires publics ou privés. 


\title{
Approximation by multipoles of the multiple acoustic scattering by small obstacles and application to the Foldy theory of isotropic scattering.
}

\author{
Abstract The asymptotic analysis, carried out in this paper, for the problem of a \\ multiple scattering of a time-harmonic wave by obstacles whose size is small as \\ compared with the wavelength establishes that the effect of the small bodies can \\ be approximated at any order of accuracy by the field radiated by point sources. \\ Among other issues, this asymptotic expansion of the wave furnishes a mathe- \\ A. Bendali \\ University of Toulouse, INSA, IMT, UMR CNRS 5219, \\ 135 avenue de Rangueil F31077, Toulouse cedex 1, France, \\ and \\ CERFACS \\ 42 Avenue Gaspard Coriolis, 31057 Toulouse Cedex 01, France, \\ Tel.: +33-5-61-55-93-32, Fax: +33-5-61-55-93-20 \\ E-mail: abendali@insa-toulouse.fr \\ P.-H. Cocquet \\ University of Genova, 2-4 rue du lièvre, CP 64, CH-1211 Genève \\ E-mail: Pierre-Henri.Cocquet@unige.ch \\ S. Tordeux \\ Project-Team Magique 3D, INRIA and University of Pau, Avenue de l'Université, BP 1155, \\ 64013 Pau, France \\ E-mail: sebastien.tordeux@inria.fr
}


matical justification with optimal error estimates of Foldy's method that consists in approximating each small obstacle by a point isotropic scatterer. Finally, it is shown how this theory can be further improved by adequately locating the center of phase of the point scatterers and taking into account of self-interactions.

\section{Introduction}

An extensive description of the numerous physical and technological issues which can be reduced to the solution of a multiple scattering problem involving scatterers, small comparatively with the wavelength, is given in [26, Chap. 7] and in [7, 17]. Solving such kinds of problems is also basic in inverse scattering [32] and in time-reversal imaging applications (cf. [21] and the references therein). Foldy's model is a simplified way for dealing with the small obstacles [14]. A comprehensive account of this approach can be found in [22, p. 297]. For the scalar wave equation, it mainly consists in characterizing the scattering properties of each of the small inclusions in the low-frequency limit by a parameter, called its scattering coefficient, and in viewing the field it scatters as the one radiated by a monopole placed at a chosen center of phase. The strengths of the equivalent monopoles are then determined by solving the corresponding scattering problem. One speaks then of 'isotropic scattering' [14]. As pointed out in [22, p. 302], the 'important word' here is 'isotropic'. For instance, such an approach does not cover the case of sound-hard scatterers $[22$, p. 302]. In this case, it is necessary to modify the usual Foldy method and to add a dipole field to correctly approximate the wave scattered by the small body [22, p. 302]. Recently, it has been observed that adding a dipole field, and thus departing from a plain isotropic scattering approach, increases the accuracy of the method notably [21].

As brought out in many studies (among many other sources, one can cite [22, ch. 8] or [26, ch. 7] and the references therein), the key property at the basis of the above reduced models lies in the fact that the wave is not really propagative at the scale of the small obstacles and as a result is governed by another model of propagation of waves: the long-wave (also called low-frequency) regime, the overall scattering problem meanwhile remaining posed in terms of the usual wave equation. When the aim is just to perform a model reduction, the link between the 
long-wave and the propagation regime is obtained by approximating the wave in the proximity of each small obstacle using one of the following techniques. The simplest of these is based on a low-frequency approximation of the wave in the immediate proximity of each small scatterer [22, Chap. 8]. Other more involved procedures use the approximation of the wave from an integral representation [21, 26] or a modal representation [7] of it again near the small obstacle.

Our first objective in this paper is to achieve a full asymptotic analysis of the multiple scattering problem to theoretically establish that such kinds of approximations can be performed at any order of accuracy. Namely the wave can be approximated at any chosen order of accuracy by accordingly increasing the order of the multipoles accounting for the field scattered by each of the small obstacles. Seemingly the approaches cited above are not adapted for this task. The main reason lies in the fact that the long-wave model must be improved when increasing the order of the asymptotic expansion. The only technique, apparently fulfilling such a requirement, and being used here, is the method of matched asymptotic expansions (see, e.g., [11-13]).

This asymptotic analysis is first used to prove optimal error estimates for Foldy's usual isotropic model. The justification of this model has been already obtained by other techniques. One of these is based on integral equations methods (see, e.g., $[28,27,26,8])$. Another method for performing this justification, limited to disks in $2 \mathrm{D}$ and spheres in $3 \mathrm{D}$, is to use a modal expansion of the wave around each small object [7]. Actually, the real novelty and the importance of the asymptotic expansion approach lie in the possibility to improve the intuitive Foldy model. This expansion shows how it is possible to gain one further order of convergence by adding a term accounting for the self-interaction effects, as recently pointed out by Liao and Ji [21] in another context, and more importantly by introducing a correction to the centers of phase of the corresponding monopoles. Such a correction, which is irrelevant when the considered scatterers are spheres [21] or disks [7] does not seem to have been proposed before, even in an heuristic way.

The geometry of the small obstacles considered in this study is quite general. They are only assumed to be Liptschitz. However, to improve the readibility, we limit ourselves to the case where the scattering properties of each of them are char- 
acterized either by a surface impedance, a sound-hard or a sound-soft boundary condition. The considered surface impedance, also termed sometimes a wall-law, can be seen as critical in terms of the transition from a sound-hard to a sound-soft obstacle. Some indications will be also given concerning the appropriate adaptations needed for dealing with penetrable obstacles and the difficulties steming from other types of impedance boundary conditions.

The outline of the paper is as follows. In Section 2, after giving the statement of the scattering problem, we define the method of matched asymptotic expansions used to expand the total wave. In particular, we set out the matching rules used to link the expansions of the propagative part of the wave and its small scale components in the proximity of the small obstacles. Section 3 is dedicated to the proof of the existence and uniqueness of the two-scale asymptotic expansions. It is at this level that is established the fact that the expansion of the propagative part of the wave consists of multipoles, i.e., spherical wavefunctions [22, p. 69]. A serious difficulty stems then: there are many ways to write down these wavefunctions. Fortunately enough, the power series expansion of the spherical Hankel establishes that ultimately any multipole can be unambiguously identified. Section 4 is dedicated to give a concrete and rigorous shape to the previous matched asymptotic expansions. A uniformely valid approximation of the total wave is constructed and validated by means of error estimates. It is next shown how it can be used to get optimal error estimates for the corresponding approximation of the propagative part of the wave. These expansions of the wave are next used in section 5 first to retrieve the usual Foldy method of isotropic scattering, to establish an optimal error bound for the corresponding approximation, and next to improve it through the procedures mentioned above. In the final Section, we discuss whether the techniques developed in this study can or cannot handle some other kinds of scattering problems.

\section{The Matched asymptotic expansions method}

In this section, we consider the scattering problem dealt with in this study. We also detail the method of matched asymptotic expansions used to get an asymptotic expansion of the total wave at any order of accuracy. 


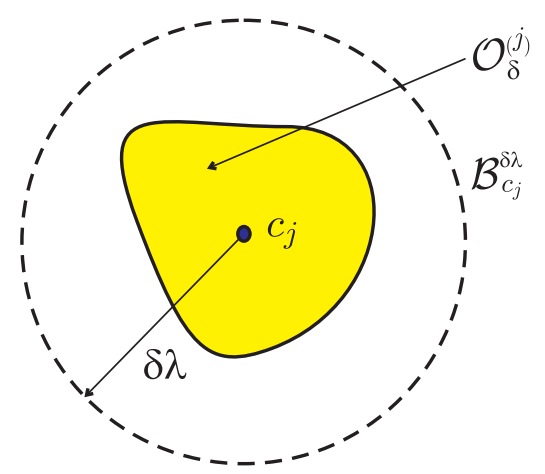

Fig. 1 Geometry of the inhomogneneities

2.1 The multiple scattering problem

We denote by $\kappa$ and $\lambda$ the wave number and the wavelength respectively in the infinite medium of propagation. Each of these is defined in terms of the other from the relation $\kappa \lambda=2 \pi$. Any of the small obstacles is an open domain $\mathscr{O}_{\delta}^{(j)}$ strictly contained in $\mathscr{B}_{c_{j}}^{\delta \lambda}$ (i.e. $\overline{\mathscr{O}_{\delta}^{(j)}} \subset \mathscr{B}_{c_{j}}^{\delta \lambda}(j=1, \ldots, N)$ ) where $\mathscr{B}_{c_{j}}^{\delta \lambda}$ is the open ball of $\mathbb{R}^{3}$ centered at $c_{j}$ and of radius $\delta \lambda$ (see figure 1). The dimensionless parameter $\delta>0$ is used to state that the obstacles are of small size as compared with the wavelength by assuming that

$$
\delta \ll 1
$$

As regards the geometrical smoothness of the scatterers, they are implicitly assumed to be at least Lipschitz.

The $c_{j}$ are the 'psychological' positions of the small scatterers, i.e. the locations of the small scatterers when observed at a sufficiently large distance or at large scale. At the end of this study, we will develop a rigorous approach for settling the position of the $c_{j}$ which are taken as the centers of phase of the point scatterers in Foldy's model.

At this point, it is necessary to clarify the asymptotic regime that is considered. Four parameters enter into the picture in this respect: the wavelength $\lambda$, the size of the small objects characterized above by the small parameter $\delta$, the smallest distance separating each pair of heterogeneities which can be linked to the wave- 
length by means of a positive parameter $d$ as follows

$$
\min _{1 \leq i, j \leq N}\left|c_{i}-c_{j}\right| \geq d \lambda,
$$

and the number $N$ of particules. In this study, all of these parameters are assumed to be fixed except the size of the scatterers which goes to 0 as $\delta \rightarrow 0$.

The multiple scattering problem can then be stated as follows in terms of the total wave $u_{\delta}$

$$
\left\{\begin{array}{l}
\left(\Delta+\kappa^{2}\right) u_{\delta}=0 \text { in } \Omega_{\delta} \\
u_{\delta}(x)=u_{\text {inc }}(x)+\frac{\exp (i \kappa|x|)}{|x|} a_{\delta}(x /|x|)+\underset{|x| \rightarrow \infty}{o}(1 /|x|),
\end{array}\right.
$$

supplemented with one of the following boundary condition

- impedance boundary condition

$$
\partial_{\mathbf{n}_{j}} u_{\delta}=\frac{v_{j}}{\delta} u_{\delta} \text { on } \partial \mathscr{O}_{\delta}^{(j)}(j=1, \ldots, N),
$$

- sound-soft obstacle

$$
u_{\delta}=0 \text { on } \partial \mathscr{O}_{\delta}^{(j)}(j=1, \ldots, N)
$$

- sound-hard obstacle

$$
\partial_{\mathbf{n}_{j}} u_{\delta}=0 \text { on } \partial \mathscr{O}_{\delta}^{(j)}(j=1, \ldots, N) .
$$

The data and the notation are the following.

- The infinite domain in which is set the scattering problem (see Fig. 2) is denoted by

$$
\Omega_{\delta}=\mathbb{R}^{3} \backslash\left(\bigcup_{j=1}^{N} \overline{\mathscr{O}_{\delta}^{(j)}}\right)
$$

- Coefficient $v_{j}$ characterizes the scattering properties of small obstacle $\mathscr{O}_{\delta}^{(j)}$ through an impedance condition set on its boundary $\partial \mathscr{O}_{\delta}^{(j)}$. Assumption

$$
\mathfrak{I} v_{j}<0
$$

is used to characterize its absorption of the incident wave acoustic energy (cf., e.g. [18])

- The unit normal $\mathbf{n}_{j}$ on $\partial \mathscr{O}_{\delta}^{(j)}$ is directed outwards $\mathscr{O}_{\delta}^{(j)}$. 


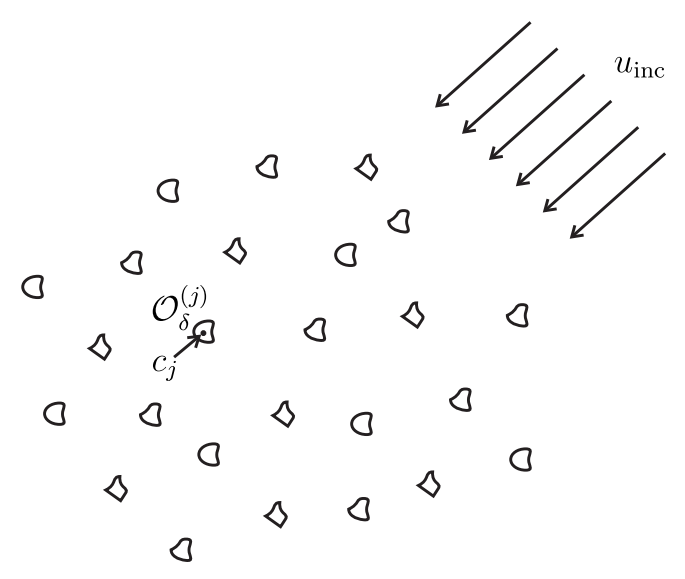

Fig. 2 Schematic view of the scattering problem

- The incident wave is denoted by $u_{\text {inc }}$. Usually, it is a plane wave $u_{\text {inc }}(x)=$ $\exp \left(i \kappa \mathbf{d} \cdot \mathbf{r}_{x}\right)$ of complex amplitude 1 , and propagating in the direction of the unit vector $\mathbf{d}, \mathbf{r}_{x}$ being the radius vector of the point $x$.

For either of the above boundary conditions, it can then be classically established [31] that problem (3) has a unique solution $u_{\delta}$ such that $\varphi u_{\delta} \in H^{1}\left(\Omega_{\delta}\right)$ for any $\varphi \in \mathscr{D}\left(\mathbb{R}^{3}\right)$. The notation $\mathfrak{R} z$ and $\mathfrak{I} z$ stands for the real and imaginary parts respectively of the complex number $z$.

As said above, we now focus on building an asymptotic expansion for $u_{\delta}$ using the method of matched asymptotic expansions.

\subsection{The matched asymptotic expansions}

The method of matched asymptotic expansions consists here in expanding the wave in the proximity of each of the small obstacles and far enough from these and adequately linking the two expansions called inner and outer in the terminology of this approach.

\subsubsection{The inner and outer expansions}

The construction of the above expansions relies upon the introduction of a global field, called far or outer, describing the overall behavior of the field scattered out- 
side the immediate vicinity of the small scatterers and a field defined inside the boundary layer enclosing each of them, known as near or inner. The outer expansion is obtained by postulating that the far field has the following expansion:

$$
u_{\delta}(x)=\sum_{k=0}^{m} \delta^{k} u_{k}(x)+\underset{\delta \rightarrow 0}{o}\left(\delta^{m}\right), x \in \mathbb{R}^{3} \text { and } x \neq c_{j}(j=1, \ldots, N) .
$$

The near field corresponds to a zoom on each of the $\mathscr{O}_{\delta}^{(j)}$ and is expressed in terms of the fast variables

$$
X=\frac{x-c_{j}}{\delta}
$$

According to the general approach of the method of matched asymptotic expansions, we look for an expansion for the solution to the above multiple scattering problem inside the boundary layer around each small obstacle in the following form:

$$
\Pi_{\delta}^{(j)}(X)=u_{\delta}\left(c_{j}+\delta X\right)=\sum_{k=0}^{m} \delta^{k} \Pi_{k}^{(j)}(X)+\underset{\delta \rightarrow 0}{o}\left(\delta^{m}\right) .
$$

Remark 1 Few comments are in order to clarify the notation and some features of the asymptotic expansions.

1. Coordinates $X$ depend on the particular small scatterer being involved. However this dependence is left implicit for simplicity. We can also sometimes more appropriately express $X$ in terms of the spherical coordinates as $X=R \Theta$ with $R=|X|$ and $\Theta=X /|X|$.

2. Far and near field is a terminology more linked to scattering problems. Inner and outer are more usual in the framework of matched asymptotic expansions. Below we will use one or the other of these terminologies when referring to these expansions and to what concerns them such as for example the coefficients of the asymptotic expansions or the boundary-value problems these coefficients satisfy.

3. Any inner expansion 'sees' its surounding medium as if it were infinite. In mathematical words, this amounts to define the expansion on the exterior $\mathbb{R}^{3} \backslash$ $\widehat{\mathscr{O}^{(j)}}$ of a scaled description

$$
\widehat{\mathscr{O}^{(j)}}=\left\{X \in \mathbb{R}^{3} ; x=\delta X \in \mathscr{O}_{\delta}^{(j)}\right\}
$$

of the obstacle. The outer unit normal to $\partial \widehat{\widehat{O O}^{(j)}}$ is denoted by $\widehat{\mathbf{n}_{j}}$. 
4. Parameter $\delta$ is at our disposal, as soon as it tends to zero. We can thus assume that each of the scaled obstacles $\widehat{\widehat{\mathscr{O}^{(j)}}}$ is included in the unit ball $|X| \leq 1$. In this way, the geometrical data related to the inner problems when all the obstacles are balls of the same radius is simply a ball of radius $R_{0} \leq 1$. In other words, all the normalized domains $\widehat{\mathcal{O}^{(j)}}$ then coincide with the ball $|X|<R_{0} \leq 1$. (see [2]).

\subsubsection{The matching rules}

The matching conditions link the inner and the outer expansions and allow for their complete determination. Actually these conditions express what is known as the Van Dyke principle enforcing that either of these expansions corresponds to the same solution. Their specific statement depends on the problem under consideration (cf., e.g., [5,11,13,19]). For the scattering problem being considered here, we proceed as in [3]. The matching conditions are set during the determination of the expansions as follows:

- A truncated outer expansion is expressed with respect to the fast variables:

$$
\sum_{l=0}^{m} \delta^{l} u_{l}\left(c_{j}+\delta X\right)
$$

It is then expanded in powers of $\delta$ and truncated to get:

$$
\sum_{l=0}^{m} \delta^{l} u_{l}\left(c_{j}+\delta X\right)=\sum_{l=0}^{m} \delta^{l} \mathrm{U}_{m, l}^{(j)}(X)+\underset{\delta \rightarrow 0}{o}\left(\delta^{m}\right),
$$

We think that it is appropriate to refer to the $\mathrm{U}_{m, l}^{(j)}$ as the matching functions.

- The matching conditions are then set as follows:

$$
\Pi_{k}^{(j)}-\mathrm{U}_{m, k}^{(j)}=\underset{|X| \rightarrow+\infty}{o}\left(\frac{1}{|X|^{m-k}}\right)(k=0, \ldots, m, j=1, \ldots, N) .
$$

Hereafter, the convergence corresponding to $o_{|X| \rightarrow+\infty}$ or to $O_{|X| \rightarrow+\infty}$ is always assumed to be uniform relatively to $X$.

It is worth noting that assuming an expansion for $\sum_{l=0}^{m} \delta^{l} u_{l}\left(c_{j}+\delta X\right)$ in the form (11) implies that the outer coefficients $u_{k}$ have a singularity of finite order at each $c_{j}$, i.e., there exist $\gamma>0, \rho>0$ and $M$ such that

$$
\left|x-c_{j}\right|^{\gamma}\left|u\left(x-c_{j}\right)\right| \leq M \text { for }\left|x-c_{j}\right| \leq \rho .
$$




\section{Existence and uniqueness of the asymptotic expansions at any order}

In this section, we prove that the inner and the outer coefficients can be determined at any order in a unique way.

\subsection{The inner and outer problems}

Plugging expansion (9) into system (3) and identifying terms corresponding to the same power of $\delta$, we get that each coefficient of the outer expansion satisfies:

$$
\left\{\begin{array}{l}
\left(\Delta+\kappa^{2}\right) u_{k}(x)=0, x \in \mathbb{R}^{3} \text { and } x \neq c_{j}(j=1, \ldots, N), \\
\lim _{|x| \rightarrow+\infty}|x|\left(\partial_{|x|}-i \kappa\right)\left(u_{k}-\delta_{k, 0} u_{\text {inc }}\right)=0,
\end{array}(k=0,1, \ldots)\right.
$$

where $\delta_{k, 0}$ is the Kronecker symbol: $\delta_{k, 0}=1$ if $k=0$ and 0 otherwise.

We already said that matching rules (12) compel the coefficients $u_{k}$ of the outer expansion to have a singularity of finite order at each $c_{j}$. Both the form of the expansions and the restriction assumed on the singularity of the coefficients of the outer one will be validated by error estimates ensuring the approximation of $u_{\delta}$ by an adequate combination of the truncated expansions $\sum_{k \leq m} \delta^{k} u_{k}$, and $\sum_{k \leq m} \delta^{k} \sum_{0 \leq j \leq N} \Pi_{k}^{(j)}$.

Proceeding in the same way as for the far fields, we plug expansion (10) into (3) and identify terms with the same powers of $\delta$. We then get the following kind of recursive Laplace equations satisfied by the inner coefficients:

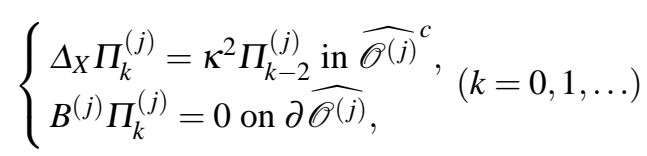

where operator $B^{(j)}$ is defined according to the boundary condition being considered

$$
B^{(j)} \Pi_{k}^{(j)}= \begin{cases}\partial_{\widehat{\mathbf{n}_{j}}} \Pi_{k}^{(j)}-v_{j} \Pi_{k}^{(j)}, & \text { for (4), } \\ \Pi_{k}^{(j)}, & \text { for (5), } \\ \partial_{\widehat{\mathbf{n}_{j}}} \Pi_{k}^{(j)}, & \text { for (6), }\end{cases}
$$

and ${\widehat{\mathscr{O}^{(j)}}}^{c}=\mathbb{R}^{3} \backslash \widehat{\widehat{\mathcal{O}^{(j)}}}$ stands for the open complement of $\mathscr{O}^{(j)}$. Any term $\Pi_{\ell}^{(j)}$ corresponding to a subscript $\ell<0$ is implicitly assumed to be zero. Condition $\mathfrak{I} v_{j}<0$ simplifies some stability results. 
Remark 2 The above recursive Laplace equations is a sharper way, improving with the order of the expansion, to account for the long-wave regime in the vicinity of the small obstacles. The crudest model simply consists in discarding any propagation effect.

The remainder of this section is dedicated to prove the following theorem.

Theorem 1 Above equations (14), (15) and matching conditions defined through (11) and (12) recursively determine one and only one system of inner and outer coefficients $\left(u_{k}, \Pi_{k}^{(j)}\right)$ for $k=0,1, \ldots$

Theorem 1 is proved by induction. It will be established for $k=0$ and for $k+1$ assuming that it is true up to order $k$. We begin with some preliminary results.

\subsection{Preliminary results}

At first, we give a complete characterization of the outer coefficients. We next do the same for the inner ones when $|X|$ is large enough. We finally establish some general properties owned by these two kinds of coefficients.

\subsubsection{General form of the outer coefficients}

The following theorem reduces the determination of the outer coefficients to that of a finite number of parameters and is proved in [2].

Theorem 2 Any solution to (14) which has a finite order of singularity as stated in (13) is in the following form:

$$
\begin{aligned}
u_{k}(x)=\sum_{j=1}^{N} \sum_{n \geq 0}^{\prime} h_{n}^{(1)}\left(\kappa\left|x-c_{j}\right|\right) Y_{n, k}^{(j)}\left(\frac{x-c_{j}}{\left|x-c_{j}\right|}\right), & \\
& x \in \mathbb{R}^{3} \text { and } x \neq c_{j}(j=1, \ldots, N)(k=0,1, \ldots) .
\end{aligned}
$$

As usual, $h_{n}^{(1)}$ stands for the spherical Hankel function of the first kind of order $n$ and $Y_{n, k}^{(j)}$ for a spherical harmonic function spanned by the $2 n+1$ spherical harmonics $Y_{n}^{m}(-n \leq m \leq n)$ of order $n$ (cf., e.g., $\left.[15,22]\right)$. The quote in $\sum_{n \geq 0}^{\prime}$ indicates that only a finite number of terms in the sum are not zero. 
The following result is fundamental for the determination of the inner coefficients.

Lemma 1 Any solution to the recursive Laplace equations

$$
\Delta_{X} \Phi_{k}^{(j)}=\kappa^{2} \Phi_{k-2}^{(j)} \text { in } \widehat{\mathscr{O}(j)}^{c},(k=0,1,2, \ldots),
$$

with $\Phi_{-1}^{(j)}=\Phi_{-2}^{(j)}=0$ and $\Phi_{k}^{(j)}$ growing at most as $|X|^{v}$ as $|X| \rightarrow \infty$ can be written as follows:

$$
\begin{aligned}
& \left.\Phi_{k}^{(j)}(X)\right|_{X=R \Theta}= \\
& \quad \sum_{n \geq 0}\left(\sum_{l=0}^{\lfloor k / 2\rfloor} c_{l, n, k}^{(j)} R^{-(n+1)+2 l}+d_{l, n, k}^{(j)} R^{n+2 l}\right) Y_{n, k}^{(j)}(\Theta) \text { for }|X|>1
\end{aligned}
$$

where $\lfloor k / 2\rfloor$ is the integral part of $k / 2$ and only a finite number of coefficients $d_{l, n, k}^{(j)}$ are not zero. The series converges uniformly as well as any of its derivatives for $|X| \geq 1$. In particular, for any integer $\ell$

$$
\Phi_{k}^{(j)}(X)=\underset{|X| \rightarrow+\infty}{o}\left(|X|^{\ell}\right) \text { if and only if } \Phi_{k}^{(j)}(X)=\underset{|X| \rightarrow+\infty}{O}\left(|X|^{\ell-1}\right)
$$

and

$$
\text { if } \Phi_{k}^{(j)}(X)=\underset{|X| \rightarrow+\infty}{O}\left(|X|^{\ell}\right) \text {, then } \partial_{|X|} \Phi_{k}^{(j)}(X)=\underset{|X| \rightarrow+\infty}{O}\left(|X|^{\ell-1}\right) .
$$

Proof Expansion (19) is established in [2]. Interior elliptic estimates then readily give that $\Phi_{k}^{(j)}$ is $\mathscr{C}^{\infty}$ for $|X|>1$. The rest of the proof is a straightforward consequence of the properties of functions having a power series expansion.

Remark 3 Theorem 2 and Lemma 1 provide the core foundation for the proof of theorem 1.

\subsubsection{Useful properties of the asymptotic expansion}

We first show that the matching functions $\mathrm{U}_{m, l}^{(j)}$ satisfy a system of recursive Laplace equations.

Lemma 2 Let $m$ and $l$ be integers such that $0 \leq l \leq m$. Then the matching functions (11) are solution to the recursive Laplace equations:

$$
\Delta \mathrm{U}_{m, l}^{(j)}(X)=-\kappa^{2} \mathrm{U}_{m, l-2}^{(j)}(X) \text { for }|X| \neq 0
$$

with $\mathrm{U}_{m, l-2}^{(j)}=0$ if $l-2<0$. 
Proof Asymptotic expansions of the spherical Bessel and Neumann functions (cf., e.g., $[15,22])$ show that $u_{m, \delta}$ is a meromorphic function with a pole at $c_{j}$ thus ensuring the existence of expansions (11). Furthermore the Laurent series expansion of $u_{m, \delta}$ at $c_{j}$ can be differentiated term by term to yield

$$
\delta^{2} \Delta_{x} \sum_{l=0}^{m} \delta^{l} u_{l}\left(c_{j}+\delta X\right)=\sum_{l=0}^{m} \delta^{l} \Delta_{X} \mathrm{U}_{m, l}^{(j)}+o_{\delta \rightarrow 0}\left(\delta^{m}\right)
$$

Using the fact that $u_{m, \delta}$ satisfies the Helmholtz equation, we get

$$
\begin{aligned}
\delta^{2} \Delta_{x} \sum_{l=0}^{m} \delta^{l} u_{l}\left(c_{j}+\delta X\right) & =-\kappa^{2} \delta^{2} u_{m, \delta}\left(c_{j}+\delta X\right) \\
& =-\kappa^{2} \delta^{2} \sum_{l=0}^{m-2} \delta^{l} \mathrm{U}_{m, l}^{(j)}(X)+o_{\delta \rightarrow 0}\left(\delta^{m}\right) \\
& =-\kappa^{2} \sum_{l=2}^{m} \delta^{l} \mathrm{U}_{m, l-2}^{(j)}+o_{\delta \rightarrow 0}\left(\delta^{m}\right)
\end{aligned}
$$

The rest of the proof follows from the identification of the two expansions in a straightforward way.

Next lemma gives an accurate expression for the asymptotic behavior of $\Pi_{l}^{(j)}$ $\mathrm{U}_{k, l}^{(j)}$ as $|X| \rightarrow \infty$. This result is basic for proving that the matching conditions uniquely determine the singular parts of the outer coefficients $u_{k}$.

Lemma 3 Assume that theorem 1 is valid up to order $k$. Then

$$
\left(\mathrm{U}_{k, l}^{(j)}-\Pi_{l}^{(j)}\right)(R \Theta)=\frac{1}{R^{(k+1)-l}} \sum_{\gamma=0}^{\lfloor l / 2\rfloor} \mathscr{Y}_{2 \gamma+k-l, k, l}^{(j)}(\Theta)+\underset{R \rightarrow+\infty}{O}\left(\frac{1}{R^{k+2-l}}\right)
$$

for $|X|>1$, and $l=0, \ldots, k$, where $\mathscr{Y}_{v, k, l}^{(j)}(\Theta)$ are determined spherical harmonic functions of order $v$ satisfying the following recurrence relations:

$$
\mathscr{Y}_{2 \gamma+k-l, k, l}^{(j)}(\Theta)=\frac{\kappa^{2}}{2 \gamma(2(k+\gamma-l)+1)} \mathscr{Y}_{2 \gamma+k-l, k, l-2}^{(j)}(\Theta)(l=2, \ldots, k, \gamma=1, \ldots,\lfloor l / 2\rfloor) .
$$

Proof Define $\varphi_{k, l}=\mathrm{U}_{k, l}^{(j)}-\Pi_{l}^{(j)}(l=0, \ldots, k)$. Lemmas 2 and 15 directly yield that $\varphi_{k, l}$ also satisfy the recursive Laplace equations

$$
\Delta \varphi_{k, l}=-\kappa^{2} \varphi_{k, l-2}, \text { for }|X| \geq 1
$$


ensuring as in lemma 1 that

$$
\varphi_{k, l}(R \Theta)=\sum_{n \geq 0} \sum_{|m| \leq n}\left(\sum_{\gamma=0}^{\lfloor l / 2\rfloor} c_{\gamma, n, k, l}^{m,(j)} R^{-(n+1)+2 \gamma}+d_{\gamma, n, k, l}^{m,(j)} R^{n+2 \gamma}\right) Y_{n}^{m}(\Theta) .
$$

Matching rules (12) then yield $\varphi_{k, l}(R \Theta)=O_{R \rightarrow+\infty}\left(R^{l-k-1}\right)$. Function $\varphi_{k, l}$ is thus the sum of a term in $R^{l-(k+1)}$ and a rest given by a power series in $1 / R$ whose coefficients are spherical harmonic functions. As a result, any rest being a $o_{R \rightarrow+\infty}\left(1 / R^{t}\right)$ is actually a $O_{R \rightarrow+\infty}\left(1 / R^{t+1}\right)$. This feature will be important in a subsequent proof establishing that the coefficients of the inner expansion do exist.

The coefficient of $R^{l-(k+1)}$ can be obtained by collecting the terms corresponding to $n$ and $\gamma$ such that $-(n+1)+2 \gamma=-(k+1)+l$. The order of the corresponding spherical harmonic is thus $n=2 \gamma+k-l$ so that the term in $R^{l-(k+1)}$ involved in the expansion of $\varphi_{k, l}$ can be written as follows

$$
R^{-(k+1)+l} \sum_{\gamma=0}^{\lfloor l / 2\rfloor} \mathscr{Y}_{2 \gamma+k-l, k, l}^{(j)}(\Theta)
$$

where $\mathscr{Y}_{v, k, l}^{(j)}(\Theta)$ is a spherical harmonic function of order $v$. The first part of the lemma is therefore proved.

To prove the recurrence relation for $\mathscr{Y}_{2 \gamma+k-l, k, l}^{(j)}(\Theta)$, we use the recursive Laplace equations satisfied by $\varphi_{k, l}$. Expressing the Laplace operator in spherical coordinates, we get

$$
\begin{aligned}
R^{2} \Delta_{X} \varphi_{k, l}= & \left(\left(R \partial_{R}\right)^{2}+\left(R \partial_{R}\right)+\Delta \mathscr{S}\right) \varphi_{k, l} \\
=\frac{1}{R^{(k+1)-l}} \sum_{\gamma=0}^{\lfloor l / 2\rfloor} 2 \gamma(2(l-\gamma-k)-1) \mathscr{Y}_{2 \gamma+k-l, k, l}^{(j)}(\Theta) & +\underset{R \rightarrow+\infty}{O}\left(\frac{1}{R^{k+2-l}}\right) .
\end{aligned}
$$

We have used the following properties: $\left(R \partial_{R}\right)^{v} R^{\alpha}=\alpha^{v} R^{\alpha}$ and $\Delta_{\mathscr{S}} Y_{n}^{m}(\Theta)=$ $-n(n+1) Y_{n}^{m}(\Theta)$. Expanding $\varphi_{k, l-2}$ in the same way, we come to

$$
\begin{aligned}
& R^{2} \varphi_{k, l-2}=\frac{R^{2}}{R^{k+1-l+2}} \sum_{\gamma=0}^{\lfloor(l-2) / 2\rfloor} \mathscr{Y}_{2(\gamma+1)+k-l, k, l-2}^{(j)}(\Theta)+R^{2} \underset{R \rightarrow+\infty}{O}\left(\frac{1}{R^{k+2-l+2}}\right) \\
&=\frac{1}{R^{k+1-l}} \sum_{\gamma^{\prime}=1}^{\lfloor l / 2\rfloor} \mathscr{Y}_{2 \gamma^{\prime}+k-l, k, l-2}^{(j)}(\Theta)+\underset{R \rightarrow+\infty}{O}\left(\frac{1}{R^{k+2-l}}\right),
\end{aligned}
$$


The latter expression has been obtained by the change of the summation index $\gamma^{\prime}=$ $\gamma+1$. The recursive Laplace equations $\Delta \varphi_{k, l}=-\kappa^{2} \varphi_{k, l-2}$ finally yield recurrence formula (23).

\subsection{Proof of the existence and the uniqueness of the expansions}

As said above, we prove Theorem 1 by induction. We establish that the coefficients of both inner and outer expansions can be uniquely determined through the following procedure. We prove the theorem first for $k=0$. Assuming next that it is true up to order $k$, we prove that the $(k+1)$-th order inner coefficient is uniquely determined. We then complete the proof by establishing the same statement for the outer coefficient of order $k+1$.

\subsubsection{Zeroth-order expansions}

Noting that $h_{n}^{(1)}(z) \sim C_{n} z^{-n-1}$ for $z \rightarrow 0$ (cf., e.g., [15]), matching conditions (12) impose that $u_{0}$ is not singular in the neighborhood of any of the $c_{j}$. As a result,

$$
u_{0}=u_{\text {inc }} \text { in } \mathbb{R}^{3} .
$$

To get the zero-th order inner coefficient, we first compute the matching functions (11). A Taylor expansion yields:

$$
u_{0}\left(c_{j}+\delta R \Theta\right)=u_{0}\left(c_{j}\right)+\underset{\delta \rightarrow 0}{o}(1)=\mathrm{U}_{0,0}^{(j)}(X)+\underset{\delta \rightarrow 0}{o}(1) .
$$

Functions $\Pi_{0}^{(j)}$ then satisfy the following boundary-value problem on the open complement $\widehat{\mathscr{O}}^{c}{ }^{c}=\mathbb{R}^{3} \backslash \widehat{\widehat{\mathscr{O}^{(j)}}}$ of $\widehat{\mathscr{O}^{(j)}}$

$$
\left\{\begin{array}{l}
\Delta \Pi_{0}^{(j)}=0 \text { in } \widehat{\mathscr{O}}^{(j)} \\
B^{(j)} \Pi_{0}^{(j)}=0 \text { on } \partial \widehat{\mathscr{O}}^{(j)}, \\
\Pi_{0}^{(j)}(X)-\mathrm{U}_{0,0}^{(j)}(X)=o_{|X| \rightarrow+\infty}(1),
\end{array}\right.
$$

in which $B^{(j)}$ is the differential boundary operator defined in (16).

To solve problem (24), we consider the following one

$$
\left\{\begin{array}{l}
\Delta \Phi^{(j)}=0 \text { in }{\widehat{\mathscr{O}^{(j)}}}^{c} \\
B^{(j)} \Phi^{(j)}=0 \text { on } \partial \widehat{\mathscr{O}^{(j)}} \\
\Phi^{(j)}(X)=1+\underset{|X| \rightarrow \infty}{o}(1),
\end{array}\right.
$$


A solution to (25) is sought in the form $\Phi^{(j)}=1+\Phi_{\mathrm{BL}}^{(j)}$ where its variational part $\Phi_{\mathrm{BL}}^{(j)}$ is lying in the usual Beppo-Levi space (cf., e.g., [20,24])

$$
\mathrm{BL}^{(j)}=\left\{\Phi \in \mathscr{D}^{\prime}\left(\widehat{\mathscr{O}(j)}^{c}\right) ;\left(1+|X|^{2}\right)^{-1 / 2} \Phi \text { and } \nabla \Phi \in L^{2}\left(\widehat{\mathscr{O}}^{c}\right)\right\}
$$

since, as established in [24] for instance, this space is a Hilbert space for the norm

$$
\|\Phi\|_{\mathrm{BL}^{(j)}}=\|\nabla \Phi\|_{L^{2}\left(\widehat{\mathscr{O}}^{(j)}\right)} .
$$

We can then state the following lemma.

Lemma 4 The zero-th order inner coefficient $\Pi_{0}^{(j)}$ satisfying (24) is uniquely defined and is given by

$$
\Pi_{0}^{(j)}=u_{0}\left(c_{j}\right) \Phi^{(j)}
$$

Proof Clearly, it is enough to prove that problem (25) is well-posed in the following functional framework

$$
H_{\mathrm{loc}}^{1}\left({\widehat{\widehat{\mathscr{O}}^{(j)}}}^{c}\right)=\left\{\Phi \in \mathscr{D}^{\prime}\left({\widehat{\mathscr{O}^{(j)}}}^{c}\right) ; \theta \Phi \in H^{1}\left(\widehat{\mathscr{O}}^{c}{ }^{c}\right), \forall \theta \in \mathscr{D}\left(\mathbb{R}^{3}\right)\right\}
$$

The integral representations of the solution to the Laplace equation (cf., e.g., [16, 24]) readily yield that $\Gamma^{(j)}=1-\Phi^{(j)}$ satisfies

$$
\Gamma^{(j)}(X)=O\left(\frac{1}{|X|}\right)
$$

and thus belongs to $\mathrm{BL}^{(j)}$. The boundary condition satisfied by $\Phi^{(j)}$ on $\partial \widehat{\mathscr{O}^{(j)}}$ yields the following one for $\Gamma^{(j)}$

$$
\begin{cases}\Gamma^{(j)}=1, & \text { for } \Phi^{(j)}=0 \\ \partial_{\widehat{\mathbf{n}_{j}}} \Gamma^{(j)}-v_{j} \Gamma^{(j)}=-v_{j}, & \text { for } \partial_{\widehat{\mathbf{n}_{j}}} \Phi^{(j)}-v_{j} \Phi^{(j)}=0 \\ \partial_{\widehat{\mathbf{n}_{j}}} \Gamma^{(j)}=0, & \text { for } \partial_{\widehat{\mathbf{n}_{j}}} \Phi^{(j)}=0\end{cases}
$$

Thus, we are accordingly led to solve one of the following variational problems

$$
\begin{aligned}
& \left\{\begin{array}{l}
\Gamma^{(j)} \in \mathrm{BL}^{(j)},\left.\Gamma^{(j)}\right|_{\partial \widehat{\mathscr{O}^{(j)}}}=1, \forall \Psi \in \mathrm{BL}^{(j)},\left.\Psi\right|_{\partial \widehat{\mathscr{O}^{(j)}}}=0, \\
\int_{\widehat{\mathscr{O}}^{(j)}} c \nabla \Gamma^{(j)} \cdot \nabla \Psi d x=0,
\end{array}\right. \\
& \left\{\begin{array}{l}
\Gamma^{(j)} \in \mathrm{BL}^{(j)}, \forall \Psi \in \mathrm{BL}^{(j)}, \\
\int_{\widehat{\mathscr{O}}^{(j)}} \nabla \Gamma^{(j)} \cdot \nabla \Psi d x+v_{j} \int_{\partial \widehat{\mathscr{O}}^{(j)}} \Gamma_{0}^{(j)} \Psi d \sigma=-v_{j} \int_{\partial \widehat{\mathscr{O}^{(j)}}} \Psi d \sigma,
\end{array}\right.
\end{aligned}
$$




$$
\left\{\begin{array}{l}
\Gamma^{(j)} \in \mathrm{BL}^{(j)}, \forall \Psi \in \mathrm{BL}^{(j)} \\
\int_{\widehat{\mathscr{O}}^{(j)}} c \Gamma^{(j)} \cdot \nabla \Psi d x=0 .
\end{array}\right.
$$

Let us denote by

$$
a^{(j)}(\Phi, \Psi)=\left\{\begin{array}{l}
\int_{\widehat{\mathscr{O}^{(j)}}} c \nabla \Phi \cdot \nabla \Psi d x+v_{j} \int_{\partial \widehat{\mathscr{O}(j)}} \Phi \Psi d \sigma, \\
\int_{\widehat{\widehat{O}^{(j)}}} c \nabla \Phi \cdot \nabla \Psi d x,
\end{array}\right.
$$

the bilinear form of the variational problem being considered above. In view of (26), it is directly coercive on $\mathrm{BL}^{(j)}$ for the second case in (27). For the first one, denoting by $0<\theta<\pi$ the opposite of the argument of the complex number $v_{j}$, i.e., $v_{j}=\left|v_{j}\right| e^{-i \theta}$, we can write that

$$
e^{i \theta} a^{(j)}(\Phi, \bar{\Phi})=e^{i \theta}\|\nabla \Phi\|_{L^{2}\left(\widehat{\mathscr{O}}^{(j)}\right)}+\left|v_{j}\right|\|\Phi\|_{L^{2}\left(\partial \widehat{\mathscr{O}^{(j)}}\right)}^{2} .
$$

This shows that

$$
\left.\left|a^{(j)}(\Phi, \bar{\Phi})\right| \geq \mathfrak{I}\left(e^{i \theta} a^{(j)}(\Phi, \bar{\Phi})\right)=\sin \theta\|\nabla \Phi\|_{L^{2}\left(\widehat{\mathscr{O}}^{c}\right)^{c}}\right)
$$

and hence establishes that the bilinear form is coercive on $\mathrm{BL}^{(j)}$ in this case too. Lax-Milgram lemma then straightforwardly completes the proof.

Remark 4 For the sound-hard boundary condition $\partial_{\widehat{\mathbf{n}}_{j}} \Phi^{(j)}=0, \Gamma^{(j)}=0$ and hence $\Phi^{(j)}=1$. This feature will have an important consequence for the reduction of the multi-scattering problem by Foldy's method.

We have hence established the existence and the uniqueness of the zero-th order inner and outer expansions.

\subsubsection{First part of the inductive step: determination of the outer coefficient}

In view of Theorem 2 and the singularity of spherical Hankel $h_{n}^{(1)}(z)$ at $z=0$, it can be argued that

$$
u_{k+1}(x)=\sum_{j=1}^{N} \sum_{\gamma=0}^{k} h_{\gamma}^{(1)}\left(\kappa\left|x-c_{l}\right|\right) \mathscr{H}_{\gamma, k+1}^{(j)}(\Theta),
$$

where $\mathscr{H}_{\gamma, k+1}^{(j)}(\Theta)$ are spherical harmonic functions of order $\gamma$ not yet determined. The following remark is fundamental both in the proof by induction or for explicitly obtaining the expansion up to the first-order which will be a basic step in the mathematical justification of Foldy's model. 
Remark 5 Since $\delta^{k+1} h_{\gamma}^{(1)}(\kappa \delta R) \sim_{\delta \rightarrow 0} C_{\gamma} \delta^{k-\gamma}$, the $\mathscr{H}_{\gamma, k+1}^{(l)}(\Theta)$ can be determined recursively for $\gamma=k, k-1$, until $\gamma=0$. Moreover, at each step, only the matching functions $\mathrm{U}_{k+1, l}(l=0, \ldots, k-\gamma)$ are involved for writing down the related equations. This gives rise to the following extremely important property: it is enough to expand $\sum_{l=0}^{k+1} \delta^{l} u_{l}\left(c_{j}+\delta R \Theta\right)$ up to order $k$, i.e.

$$
\sum_{l=0}^{k+1} \delta^{l} u_{l}\left(c_{j}+\delta X\right)=\sum_{l=0}^{k} \delta^{l} \mathrm{U}_{k+1, l}^{(j)}(X)+o\left(\delta^{k}\right)
$$

instead up to order $k+1$ to determine the $\mathscr{H}_{\gamma, k+1}^{(j)}(\Theta)$.

The following lemma, establishing a link between matching functions $\mathrm{U}_{k+1, l}^{(j)}$ and $\mathrm{U}_{k, l}^{(j)}$, is the first ingredient towards proving that the outer coefficient $u_{k+1}$ can be uniquely determined.

Lemma 5 The following relations hold true

$$
\begin{array}{r}
\mathrm{U}_{k+1, l}^{(j)}(R \Theta)=\mathrm{U}_{k, l}^{(j)}(R \Theta)+\frac{1}{R^{k+1-l}} \sum_{t=0}^{\lfloor l / 2\rfloor} N_{2 t+k-l, t} \mathscr{H}_{2 t+k-l, k+1}^{(j)}(\Theta) \\
(j=1, \ldots, N ; l=0, \ldots, k)
\end{array}
$$

where the constants $N_{n, \gamma}$ are those involved in the power series expansion of the spherical Neumann function (cf. [25, formulae 10.47.4, 10.2.2])

$$
i y_{n}(\kappa z)=\frac{i \sqrt{\pi}}{2} \sum_{\gamma \geq 0} \frac{(-1)^{\gamma-(n+1)}(\kappa z / 2)^{2 \gamma-(n+1)}}{\gamma ! \Gamma\left(\gamma+\frac{1}{2}-n\right)}=\sum_{\gamma \geq 0} N_{n, \gamma} z^{2 \gamma-(n+1)} ;
$$

here $\Gamma$ denotes the usual gamma function

Proof Let $l$ be an integer such that $0 \leq l \leq k$. The matching functions are defined through the above identification (29). Writing the left-hand side of (29) as

$$
\sum_{l=0}^{k+1} \delta^{l} u_{l}\left(c_{j}+\delta X\right)=\sum_{l=0}^{k} \delta^{l} u_{l}\left(c_{j}+\delta X\right)+\delta^{k+1} u_{k+1}\left(c_{j}+\delta X\right),
$$

reduces the determination of the matching functions $\mathrm{U}_{k+1, l}^{(j)}(l=0, \ldots, k)$ to that of the terms in $\delta^{l}$ involved in the expansion of $\delta^{k+1} u_{k+1}\left(c_{j}+\delta X\right)$. It is worth noting that only the singular part of $h_{n}^{(1)}=j_{n}+i y_{n}$ contributes to these terms

$$
\begin{aligned}
\delta^{k+1} u_{k+1}\left(c_{j}+\delta R \Theta\right) & =\delta^{k+1} \sum_{\gamma=0}^{k} h_{\gamma}(\kappa \delta R) \mathscr{H}_{\gamma, k+1}^{(j)}(\Theta) \\
& =\delta^{k+1} \sum_{\gamma=0}^{k} i y_{\gamma}(\kappa \delta R) \mathscr{H}_{\gamma, k+1}^{(j)}(\Theta)+o_{\delta \rightarrow 0}\left(\delta^{k}\right) .
\end{aligned}
$$


Plugging the power series expansion of $y_{\gamma}(\kappa \delta R)$ in the above relation, we come to

$$
\delta^{k+1} u_{k+1}\left(c_{j}+\delta R \Theta\right)=\delta^{k+1} \sum_{\gamma=0}^{k} \sum_{v \geq 0} N_{\gamma, v}(\delta R)^{2 v-(\gamma+1)} \mathscr{H}_{\gamma, k+1}^{(j)}(\Theta)+o_{\delta \rightarrow 0}\left(\delta^{k}\right)
$$

Making the following change of the indices related to the double summation

$$
(t=v, l=2 v-(\gamma+1)+(k+1)=2 v+k-\gamma)
$$

and dropping any term in $\delta^{l}$ with $l>k$, we get

$$
\delta^{k+1} u_{k+1}\left(c_{j}+\delta R \Theta\right)=\sum_{l=0}^{k} \frac{\delta^{l}}{R^{k+1-l}} \sum_{t=0}^{\lfloor l / 2\rfloor} N_{2 t+k-l, t} \mathscr{H}_{2 t+k-l, k+1}^{(j)}(\Theta)+o_{\delta \rightarrow 0}\left(\delta^{k}\right) .
$$

Gathering all the above results, we readily complete the proof.

Next lemma finishes the first part of the induction step.

Lemma 6 There exists one and only one outer coefficient $u_{k+1}$ satisfying the above equations and the matching conditions.

Proof The proof reduces to establish the above statement for $\mathscr{H}_{l, k+1}^{(j)}(\Theta)$. Lemma 5 and the inductive hypothesis yield

$$
\left(\mathrm{U}_{k, l}^{(j)}-\Pi_{l}^{(j)}\right)=\frac{1}{R^{(k+1)-l}} \sum_{\gamma=0}^{\lfloor l / 2\rfloor} \mathscr{Y}_{2 \gamma+k-l, k, l}^{(j)}(\Theta)+\underset{R \rightarrow+\infty}{o}\left(\frac{1}{R^{k+1-l}}\right)
$$

implying first that

$$
\sum_{t=0}^{\lfloor l / 2\rfloor} N_{2 t+k-l, t} \mathscr{H}_{2 t+k-l, k+1}^{(j)}(\Theta)=-\sum_{\gamma=0}^{\lfloor l / 2\rfloor} \mathscr{Y}_{2 \gamma+k-l, k, l}^{(j)}(\Theta)(l=0, \ldots, k)
$$

and next that

$$
\left(\mathrm{U}_{k, l}^{(j)}-\Pi_{l}^{(j)}\right)+\frac{1}{R^{k+1-l}} \sum_{t=0}^{\lfloor l / 2\rfloor} N_{2 t+k-l, t} \mathscr{H}_{2 t+k-l, k+1}^{(j)}(\Theta)=\underset{R \rightarrow+\infty}{o}\left(\frac{1}{R^{k+1-l}}\right) .
$$

Lemma 3 ensures that

$$
\mathscr{H}_{2 \gamma+k-l, k+1}^{(j)}(\Theta)=-\frac{1}{N_{2 \gamma+k-l, \gamma}} \mathscr{Y}_{2 \gamma+k-l, k, l}^{(j)}(\Theta)(l=0, \ldots, k, \gamma=0, \ldots,\lfloor l / 2\rfloor) .
$$

This is an overdetermined system since different $(l, \gamma)$ can lead to the same value for $v=2 \gamma+k-l$. 
Fortunately enough, spherical harmonic functions $-\frac{1}{N_{2 \gamma+k-l, \gamma}} \mathscr{Y}_{2 \gamma+k-l, k, l}^{(j)}(\Theta)$ are identical as soon as they are of the same degree $v=2 \gamma+k-l$. Indeed, coefficients $N_{n, \gamma}$ are linked by the following relations

$$
N_{n, \gamma}=\frac{\kappa^{2}}{2 \gamma(2(n-\gamma)+1)} N_{n, \gamma-1}, N_{n, 0}=-\sqrt{\pi} \frac{(-1)^{n+1} \kappa^{-(n+1)} 2^{n}}{\Gamma\left(\frac{1}{2}-n\right)} .
$$

Thus

$$
\mathscr{H}_{2 \gamma+k-l, k+1}^{(j)}(\Theta)=-\frac{1}{N_{2(\gamma-1)+k-(l-1), \gamma-1}} \frac{2 \gamma(2(\gamma+k-l)+1)}{\kappa^{2}} \mathscr{Y}_{2 \gamma+k-l, k, l}^{(j)}(\Theta)
$$

which in view of (23) yields

$$
\mathscr{H}_{2 \gamma+k-l, k+1}^{(j)}(\Theta)=-\frac{1}{N_{2(\gamma-1)+k-(l-2), \gamma-1}} \mathscr{Y}_{2(\gamma-1)+k-(l-2), k, l-2}^{(j)}(\Theta) .
$$

Continuing in this way, we come to

$$
\mathscr{H}_{2 \gamma+k-l, k+1}^{(j)}(\Theta)=-\frac{1}{N_{2 \gamma+k-l, 0}} \mathscr{Y}_{2 \gamma+k-l, k, l-2 \gamma}^{(j)}(\Theta) .
$$

This shows that for any fixed $k, \mathscr{H}_{2 \gamma+k-l, k+1}^{(j)}(\Theta)$ does not depend on the particular spherical harmonic function $\mathscr{Y}_{2 \gamma+k-l, k, l}^{(j)}(\Theta)$ used to define it. This completes the proof of the lemma.

\subsubsection{Second part of the inductive step: Determination of the inner coefficient}

Any $(k+1)$-th order inner coefficient satisfies

$$
\left\{\begin{array}{l}
\Delta \Pi_{k+1}^{(j)}=-\kappa^{2} \Pi_{k-1}^{(j)} \text { in } \widehat{\mathscr{O}}^{c}{ }^{c} \\
B^{(j)} \Pi_{k+1}^{(j)}=0 \text { on } \partial \widehat{\mathscr{O}}^{(j)} \\
\Pi_{k+1}^{(j)}-\mathrm{U}_{k+1, k+1}^{(j)}=o_{R \rightarrow+\infty}(1) .
\end{array}\right.
$$

The following change of unknown $\Gamma_{k+1}^{(j)}=\Pi_{k+1}^{(j)}-\mathrm{U}_{k+1, k+1}^{(j)}$ puts the above problem in the following form

$$
\left\{\begin{array}{l}
\Delta \Gamma_{k+1}^{(j)}=F \text { in } \mathbb{R}^{3} \backslash \widehat{\widehat{\mathscr{O}^{(j)}}} \\
B^{(j)} \Gamma_{k+1}^{(j)}=g \text { on } \partial \widehat{\mathscr{O}^{(j)}} \\
\Gamma_{k+1}^{(j)}=o_{R \rightarrow+\infty}(1)
\end{array}\right.
$$

with

$$
\left\{\begin{array}{l}
g=B^{(j)} \mathrm{U}_{k+1, k+1}^{(j)} \\
F=-\kappa^{2} \Pi_{k-1}^{(j)}-\Delta \mathrm{U}_{k+1, k+1}^{(j)}
\end{array},\right.
$$


Lemma 7 Problem (31) has one and only one solution $\Gamma_{k+1}^{(j)} \in \mathrm{BL}^{(j)}$ and makes it possible to uniquely determine the $(k+1)$-th order inner coefficient as follows

$$
\Pi_{k+1}^{(j)}=\mathrm{U}_{k+1, k+1}^{(j)}+\Gamma_{k+1}^{(j)} .
$$

Proof When $B^{(j)}$ is the boundary operator corresponding to a sound-hard or an impedance boundary condition, problem (31) can be put in the following variational form

$$
\left\{\begin{array}{l}
\Gamma_{k+1}^{(j)} \in \mathrm{BL}^{(j)}, \forall \Psi \in \mathrm{BL}^{(j)}, \\
a\left(\Gamma_{k+1}^{(j)}, \Psi\right)=\int_{\partial \widehat{\mathscr{O}}(j)} g \Psi d \sigma-\int_{\widehat{\mathscr{O}}^{(j)}} c F \Psi d x,
\end{array}\right.
$$

with the bilinear form already given in (27). It thus remains to check that $F$ does define a continuous inear form on $\mathrm{BL}^{(j)}$. It is worth noting that $F=0$ for $k=0$. We can thus assume that $k \geq 1$. Lemma 2 then yields

$$
F(X)=\kappa^{2}\left(\mathrm{U}_{k+1, k-1}^{(j)}(X)-\Pi_{k-1}^{(j)}(X)\right) .
$$

It is not enough to make use of the following behavior $F(X)=o_{|X| \rightarrow \infty}\left(1 /|X|^{2}\right)$, which is a direct consequence of the inductive hypothesis. A sharper estimate is needed. Making use of the first property stated in Lemma 5, we can write $F$ in the following form

$$
F(R \Theta)=\kappa^{2}\left(\begin{array}{l}
\mathrm{U}_{k, k-1}^{(j)}(R \Theta)-\Pi_{k-1}^{(j)}(R \Theta) \\
+\frac{1}{R^{2}} \sum_{t=0}^{\lfloor(k-1) / 2\rfloor} N_{2 t+k-(k-1), t} \mathscr{H}_{2 t+k-(k-1), k+1}^{(j)}(\Theta)
\end{array}\right)
$$

which in view of (30) can be also expressed as

$$
F(R \Theta)=\kappa^{2}\left(\mathrm{U}_{k, k-1}^{(j)}(R \Theta)-\Pi_{k-1}^{(j)}(R \Theta)-\frac{1}{R^{2}} \sum_{t=0}^{\lfloor(k-1) / 2\rfloor} \mathscr{Y}_{2 t+k-(k-1), k, k-1}^{(j)}(\Theta)\right) .
$$

Sharp asymptotic behavior (22) then readily yields that for a sufficiently large $A$

$$
|F(X)| \leq \frac{C}{R^{3}}(|X| \geq A) .
$$

Expressing the following integral

$$
\int_{|X| \geq A}\left|F\left(1+|X|^{2}\right)^{1 / 2} \Psi\left(1+|X|^{2}\right)^{-1 / 2}\right| d X
$$

in terms of spherical coordinates and using Cauchy-Schwartz inequality then allow us to write

$$
\int_{|X| \geq A}|F \Psi| d X \leq C \sqrt{\int_{A}^{+\infty}\left|\frac{1}{R^{3}}\right|^{2}\left(1+R^{2}\right) R^{2} d R} \int_{|X| \geq A} \frac{|\Psi|^{2}}{1+|X|^{2}} d X .
$$


Hereafter, $C$ stands for a constant not the same in all instances. Finally, we get

$$
\left|\int_{|X| \geq A} F \Psi d X\right| \leq C\|\Psi\|_{\mathrm{BL}} .
$$

The right-hand side does define a continuous linear form on $\mathrm{BL}^{(j)}$ thus implying that problem (32) is well-posed. Since $\Pi_{l}^{(j)}(l=0, \ldots, k+1)$ satisfy the recursive Laplace equations (18), Lemma 1 ensures that

$$
\left(\mathrm{U}_{k+1, k+1}^{(j)}-\Pi_{k+1}^{(j)}\right)(X)=o_{|X| \rightarrow \infty}(1) .
$$

Clearly, the same proof can be repeated with obvious adaptations for dealing with the case where $B^{(j)}$ corresponds to a sound-soft boundary condition.

\section{Error estimates}

Error estimates, established in this section, give a rigorous framework to the above asymptotic expansions. Namely they furnish a procedure enabling the approximation of the total wave $u_{\delta}$ from the outer and the inner expansions. In this respect, the approach being used relies upon considering the so-called uniformly valid approximation of $u_{\delta}$ (cf. [11]).

\subsection{The uniformly valid approximation}

The construction of the uniformly valid approximation of $u_{\delta}$ makes use of the cut-off function

$$
\chi \in \mathscr{C}^{\infty}(\mathbb{R}), \chi(r)=\left\{\begin{array}{c}
1, r \leq 1 \\
0, r \geq 2
\end{array}, \chi^{\prime}(r) \leq 0, \text { for all } r \geq 0,\right.
$$

and is carried out as follows

$$
\begin{aligned}
u_{m, \delta}(x)=(1- & \left.\sum_{j=1}^{N} \chi_{\delta}^{(j)}(x)\right) \sum_{k=0}^{m} \delta^{k} u_{k}(x) \\
& +\left(\sum_{j=1}^{N} \chi_{\delta}^{(j)}(x)\right) \sum_{k=0}^{m} \delta^{k} \Pi_{k}^{(j)}\left(\left(x-c_{j}\right) / \delta\right)\left(x \in \Omega_{\delta}\right),
\end{aligned}
$$

where $\chi_{\delta}^{(j)}(x)=\chi\left(\left|x-c_{j}\right| / \sqrt{\delta}\right)$. 
As a result, the obtained approximation coincides with the truncated inner and outer expansions of the field respectively inside and outside appropriate neighborhoods of the small obstacles

$$
\begin{aligned}
& u_{m, \delta}(x)=\sum_{k=0}^{m} \delta^{k} u_{k}(x)\left(\min _{j=1, \ldots, N}\left|x-c_{j}\right| \geq 2 \sqrt{\delta}\right), \\
& u_{m, \delta}(x)=\sum_{k=0}^{m} \delta^{k} \Pi_{k}^{(j)}\left(\frac{x-c_{j}}{\delta}\right)\left(\min _{j=1, \ldots, N}\left|x-c_{j}\right| \leq \sqrt{\delta}\right) .
\end{aligned}
$$

In the matching zones

$$
\left\{\mathscr{M}_{\delta}^{(j)}=\left\{x \in \mathbb{R}^{3}|\sqrt{\delta}<| x-c_{j} \mid \leq 2 \sqrt{\delta}\right\}\right.
$$

both expansions contribute to approximate $u_{\delta}$.

\subsection{Stability estimates}

A quite natural way for obtaining an estimate of $u_{\delta}-u_{m, \delta}$ is first to truncate the unbounded domain $\Omega_{\delta}$. The part of $u_{\delta}$ defined on the truncated zone is next taken into account through a transparent boundary condition on the fictitious boundary so introduced. This condition is expressed in terms of a Dirichlet-to-Neumann (DtN) operator defined below. An error estimate will then result from a stability property and a consistency estimate consisting of a bound on the related residual (cf., e.g., [1,4] for more details on this approach).

We begin with the truncation procedure. Let $\Upsilon$ be a connected bounded open set of $\mathbb{R}^{3}$, with smooth boundary whose outward unitary normal is $\mathbf{n}$, enclosing all the small obstacles. The DtN operator $T$ associated with the open complement $\Upsilon^{c}=\mathbb{R}^{3} \backslash \bar{\Upsilon}$ of $\Upsilon$ is defined as follows: For any given $\phi$ in $H^{\frac{1}{2}}(\partial \Upsilon)$, let $v$ be the solution to the following boundary value problem whose existence and uniqueness can be obtained for instance from the limiting-absorption principle (see, e.g., [31])

$$
\left\{\begin{array}{c}
v \in \mathscr{D}^{\prime}\left(\Upsilon^{c}\right), \theta v \in H^{1}\left(\Upsilon^{c}\right) \forall \theta \in \mathscr{D}\left(\mathbb{R}^{3}\right), \\
\left(\Delta+\kappa^{2}\right) v=0 \text { in } \Upsilon^{c}, \\
v=\phi \text { on } \partial \Upsilon \\
\lim _{|x| \rightarrow+\infty}|x|\left(\partial_{|x|}-i \kappa\right) v=0, \\
T \phi=\partial_{\mathbf{n}^{c} v} \in H^{-\frac{1}{2}}(\partial \Upsilon),
\end{array}\right.
$$


where $\mathbf{n}^{c}$ is the unit normal to $\partial \Upsilon$ pointing outwards $\Upsilon^{c}$. The coercivity properties of this operator are stated as follows. There exists a bounded linear operator $T_{0}$ acting from $H^{1 / 2}(\partial \Upsilon)$ into $H^{-1 / 2}(\partial \Upsilon)$ such that $T-T_{0} \in \mathscr{L}\left(H^{1 / 2}(\partial \Upsilon), H^{5 / 2}(\partial \Upsilon)\right)$ and

$$
\exists \gamma>0:\left\langle T_{0} v, \bar{v}\right\rangle_{H^{-1 / 2}(\Upsilon), H^{1 / 2}(\Upsilon)} \geq \gamma\|v\|_{H^{-1 / 2}(\Upsilon)}^{2}, \forall v \in H^{1 / 2}(\Upsilon) .
$$

We denote by

$$
e_{\delta, m}=u_{m, \delta}-u_{\delta}
$$

the error corresponding to the uniformly valid approximation of $u_{\delta}$. Plugging it in (3) and according to the considered boundary condition in (4), (5) or (6) respectively, we come to:

$$
\left\{\begin{array}{l}
\left(\Delta+\kappa^{2}\right) e_{\delta, m}=\left(\Delta+\kappa^{2}\right) u_{m, \delta} \text { in } \Upsilon_{\delta}=\Upsilon \backslash \overline{\bigcup_{j=1}^{N} \mathscr{O}_{\delta}^{(j)}}, \\
B^{(j)} e_{\delta, m}=0 \text { on } \partial \mathscr{O}_{\delta}^{(j)}(j=1, \ldots, N), \\
\partial_{\mathbf{n}} e_{\delta, m}+T e_{\delta, m}=0 \text { on } \partial \Upsilon,
\end{array}\right.
$$

where $\mathbf{n}$ still denotes the unit normal pointing outwards $\Upsilon$. The above problem can be equivalently expressed in the following variational form

$$
\left\{\begin{array}{l}
e_{\delta} \in V_{\delta}, \forall v \in V_{\delta} \\
a_{\delta}\left(e_{\delta}, v\right)=l_{\delta}(v),
\end{array}\right.
$$

with the following notation

$$
\begin{aligned}
& a_{\delta}(u, v)=\int_{\Upsilon_{\delta}}\left(\nabla u \cdot \nabla v-\kappa^{2} u v\right) d x+\beta_{\delta}(u, v)+\langle T u, v\rangle_{H^{-1 / 2}(\Upsilon), H^{1 / 2}(\Upsilon)}, \\
& l_{\delta} v=-\int_{\Upsilon_{\delta}}\left(\Delta u_{m, \delta}+\kappa^{2} u_{m, \delta}\right) v d x,
\end{aligned}
$$

where

$$
\beta_{\delta}(u, v)=-\sum_{j=1}^{N} \frac{v_{j}}{\delta} \int_{\partial \mathscr{O}_{\delta}^{(j)}} u v d s
$$

for the impedance condition, $\beta_{\delta}=0$ otherwise and

$$
V_{\delta}=\left\{v \in H^{1}\left(\Upsilon_{\delta}\right) ;\left.v\right|_{\partial \mathscr{O}_{\delta}^{(j)}}=0,(j=1, \ldots, N)\right\}
$$

for the sound-soft boundary condition, $V_{\delta}=H^{1}\left(\Upsilon_{\delta}\right)$ otherwise.

One main ingredient for estimating $e_{m, \delta}$ is provided by the following stability theorem. 
Theorem 3 There exists a constant $C$ independent of $\delta$ such that

$$
\left\|e_{\delta, m}\right\|_{H^{1}\left(\Upsilon_{\delta}\right)} \leq C\left\|\left(\Delta+\kappa^{2}\right) u_{m, \delta}\right\|_{L^{2}\left(\Upsilon_{\delta}\right)}
$$

Proof The estimate is a direct consequence of the following uniform inf-sup condition

$$
\|u\|_{H^{1}\left(\Upsilon_{\delta}\right)} \leq C \sup _{v \in V_{\delta}} \frac{\left|a_{\delta}(u, v)\right|}{\|v\|_{H^{1}\left(\Upsilon_{\delta}\right)}}
$$

which we prove by contradiction below. We first deal with the case of the impedance boundary condition. So assume that there exist a sequence $\delta_{n} \searrow 0$ and $u_{n} \in V_{\delta_{n}}$ such that

1. $\left\|u_{n}\right\|_{H^{1}\left(\Upsilon_{\delta_{n}}\right)}=1$,

2. $\lim _{n \rightarrow 0} a_{\delta_{n}}\left(u_{n}, v_{n}\right)=0$ for all sequences $\left(v_{n}\right)_{n \geq 0}$ such that $v_{n} \in V_{\delta_{n}}$ and $\left\|v_{n}\right\|_{H^{1}\left(\Upsilon_{\delta_{n}}\right)}=1$.

Two main arguments will lead to a contradiction. The first one is provided in [23]. It ensures that $u_{n}$ can be extended to a function $\widetilde{u}_{n} \in H^{1}(\Upsilon)$ in a stable way, i.e. $\widetilde{u}_{n} \mid r_{\delta_{n}}=u_{n}$ and

$$
\left\|u_{n}\right\|_{H^{1}\left(\Upsilon_{\delta_{n}}\right)} \leq\left\|\widetilde{u}_{n}\right\|_{H^{1}(\Upsilon)} \leq C\left\|u_{n}\right\|_{H^{1}\left(\Upsilon_{\delta_{n}}\right)},
$$

with $C$ a constant independent of $n$. The second one is the well-known fact that the subspace $\mathscr{V}$ of those $v \in H^{1}(\Upsilon)$ satisfying $v=0$ in a neighborhood of each $c_{j}$ is dense in $H^{1}(\Upsilon)$ (cf., e.g., [29]). From the weak compactness of the unit ball of Hilbert spaces, we can assume that $\lim _{n \rightarrow \infty} \widetilde{u}_{n}=u$ weakly in $H^{1}(\Upsilon)$. From the second of the two points listed above, we then easily deduce that $u$ is solution to the following problem

$$
\left\{\begin{array}{l}
u \in H^{1}(\Upsilon), \\
\Delta u+\kappa^{2} u=0 \text { in } \Upsilon, \\
\partial_{\mathbf{n}} u+T u=0 \text { on } \partial \Upsilon .
\end{array}\right.
$$

This problem is an equivalent formulation of a problem satisfying the Helmholtz equation in all of $\mathbb{R}^{3}$ and the Sommerfeld radiation condition. As well-known (cf., e.g., [31]), Rellich's lemma directly implies that $u=0$. The next ingredient is obtained by passing to the imaginary part of $a_{\delta_{n}}\left(u_{n}, \overline{u_{n}}\right)$ after noting that 


$$
\begin{aligned}
\mathfrak{I}\left\langle T u_{n}, \overline{u_{n}}\right\rangle_{H^{-1 / 2}(\Upsilon), H^{1 / 2}(\Upsilon)}= & \mathfrak{I}\left\langle\left(T-T_{0}\right) u_{n}, \overline{u_{n}}\right\rangle_{H^{-1 / 2}(\Upsilon), H^{1 / 2}(\Upsilon)} \\
\mathfrak{I} a_{\delta_{n}}\left(u_{n}, \overline{u_{n}}\right) & =\mathfrak{I}\left\langle\left(T-T_{0}\right) u_{n}, \overline{u_{n}}\right\rangle_{H^{-1 / 2}(\Upsilon), H^{1 / 2}(\Upsilon)} \\
& +\sum_{j=1}^{N} \frac{\mathfrak{I} v_{j}}{\delta_{n}} \int_{\partial \mathscr{O}_{\delta_{n}}^{(j)}}\left|u_{n}\right|^{2} d s .
\end{aligned}
$$

Since by compacity the first term of the above sum tend to 0 , using once more the second of the above assumptions, it can be argued that

$$
\lim _{n \rightarrow \infty} \frac{1}{\delta_{n}} \int_{\partial \mathscr{O}_{\delta_{n}}^{(j)}}\left|u_{n}\right|^{2} d s=0 .
$$

The contradiction is then a straightforward consequence of the fact that $a_{\delta_{n}}\left(u_{n}, \overline{u_{n}}\right)$ can thus be decomposed in a term which can be uniformly bound below by $\left\|u_{n}\right\|_{H^{1}}^{2}\left(r_{\delta_{n}}\right)$ and a rest tending to zero.

This proof does not cover the case of sound-hard or sound-soft obstacles. However, it can be obtained in the same way just by remarking that there is no integral on $\partial \mathscr{O}_{\delta}^{(j)}$ then.

We are thus led to get a bound for $\left(\Delta+\kappa^{2}\right) u_{m, \delta}$. Since $\Delta(f g)=f \Delta g+g \Delta f+$ $2 \nabla f \cdot \nabla g$, this term can be written as

$$
\begin{aligned}
\left(\Delta+\kappa^{2}\right) u_{m, \delta} & =\left(1-\sum_{j=1}^{N} \chi_{\delta}^{(j)}(x)\right)\left(\sum_{k=0}^{m} \delta^{k}\left(\Delta+\kappa^{2}\right) u_{k}(x)\right) \\
& +\left(\sum_{j=1}^{N} \chi_{\delta}^{(j)}(x)\right) \sum_{k=0}^{m} \delta^{k}\left(\Delta+\kappa^{2}\right) \Pi_{k}^{(j)}\left(\frac{x-c_{j}}{\delta}\right) \\
& +\left(\sum_{j=1}^{N} \Delta \chi_{\delta}^{(j)}(x)\right)\left(\sum_{k=0}^{m}\left(\Pi_{k}^{(j)}\left(\frac{x-c_{j}}{\delta}\right)-u_{k}(x)\right)\right) \\
& +2 \sum_{j=1}^{N} \sum_{k=0}^{m} \nabla \chi_{\delta}^{(j)}(x) \cdot \nabla\left(\Pi_{k}^{(j)}\left(\frac{x-c_{j}}{\delta}\right)-u_{k}(x)\right) .
\end{aligned}
$$

Since the inner coefficients are solution to recursive Laplace equations, we get

$$
\begin{aligned}
\left(\Delta+\kappa^{2}\right) u_{m, \delta} & =\kappa^{2} \sum_{j=1}^{N} \chi_{\delta}^{(j)}(x) \sum_{k=0}^{m} \delta^{k}\left(\Pi_{k}^{(j)}\left(\frac{x-c_{j}}{\delta}\right)-\frac{1}{\delta^{2}} \Pi_{k-2}^{(j)}\left(\frac{x-c_{j}}{\delta}\right)\right) \\
& +\sum_{j=1}^{N} \Delta \chi_{\delta}^{(j)}(x)\left(\sum_{k=0}^{m} \delta^{k}\left(\Pi_{k}^{(j)}\left(\frac{x-c_{j}}{\delta}\right)-u_{k}(x)\right)\right) \\
& +2 \sum_{j=1}^{N} \sum_{k=0}^{m} \delta^{k} \nabla \chi_{\delta}^{(j)}(x) \cdot \nabla\left(\Pi_{k}^{(j)}\left(\frac{x-c_{j}}{\delta}\right)-u_{k}(x)\right)
\end{aligned}
$$


where $\Pi_{-2}^{(j)}=\Pi_{-1}^{(j)}=0$. In previous formula, the first term is called the inner error and the last two ones the matching error. We now bound each of these one by one.

4.3 Bounds for the inner and matching errors

The bound for the inner error is established in the following lemma.

Lemma 8 Let $\mathscr{I}_{\delta}^{(j)}=\left(\mathbb{R}^{3} \backslash \mathscr{O}_{\delta}^{(j)}\right) \cap \mathscr{B}_{c_{j}}^{\sqrt{\delta}}$. The following bound

$$
\sum_{j=1}^{N}\left\|\chi_{\delta}^{(j)}\left(\sum_{k=0}^{m} \delta^{k}\left(\Pi_{k}^{(j)}-\frac{1}{\delta^{2}} \Pi_{k-2}^{(j)}\right)\right)\right\|_{L^{2}\left(\mathscr{J}_{\delta}^{(j)}\right)} \leq C \delta^{m / 2+1 / 4},
$$

holds true with a constant $C>0$ independent of $\delta$.

Proof At first, we write the sum involved in the inner error a bit differently

$$
\sum_{k=0}^{m} \delta^{k}\left(\Pi_{k}^{(j)}-\frac{1}{\delta^{2}} \Pi_{k-2}^{(j)}\right)=\delta^{m} \Pi_{m}^{(j)}+\delta^{m-1} \Pi_{m-1}^{(j)} .
$$

Triangular inequality then yields

$$
\left\|\chi_{\delta}^{(j)} \sum_{k=0}^{m} \delta^{k}\left(\Pi_{k}^{(j)}-\frac{1}{\delta^{2}} \Pi_{k-2}^{(j)}\right)\right\|_{L^{2}\left(\mathscr{J}_{\delta}^{(j)}\right)} \leq C \sum_{l=m-1}^{m} \delta^{l}\left\|\Pi_{l}^{(j)}\right\|_{L^{2}\left(\mathscr{J}_{\delta}^{(j)}\right)} .
$$

Passing to the fast variable $X=\left(x-c_{j}\right) / \delta$, we obtain:

$$
\left\|\Pi_{l}^{(j)}\right\|_{L^{2}\left(\mathscr{I}_{\delta}^{(j)}\right)}^{2}=\delta^{3} \int_{\widehat{\mathscr{I}_{\delta}^{(j)}}}\left|\Pi_{l}^{(j)}(X)\right|^{2} d X
$$

where $\widehat{\mathscr{I}_{\delta}^{(j)}}=\left({\widehat{\mathcal{O}^{(j)}}}^{c}\right) \cap \mathscr{B}_{0}^{1 / \sqrt{\delta}}$. Lemmas 4 and 7 yield

$$
\Pi_{l}^{(j)}(X)=\mathrm{U}_{l, l}^{(j)}(X)+\Gamma_{l}^{(j)}(X)\left(X \in \widehat{\mathscr{O}}^{c(j)}\right),
$$

where $\mathrm{U}_{l, l}^{(j)}$ and $\Gamma_{l}^{(j)}$ are respectively the matching function and the solution to variational problem (32) introduced above. With the notation introduced there, one thus obtains the bound

$$
\begin{aligned}
\left\|\Gamma_{l}^{(j)}\right\|_{L^{2}\left(\widehat{\mathscr{J}}_{\delta}^{(j)}\right)} & =\left(\int_{\widehat{\mathscr{I}_{\delta}^{(j)}}}\left|(1+|X|)^{-1 / 2} \Gamma_{l}^{(j)}(X)\right|^{2}(1+|X|) d X\right)^{1 / 2} \\
& \leq\left(1+\frac{1}{\sqrt{\delta}}\right)^{1 / 2}\left\|\left(1+|X|^{2}\right)^{-1 / 2} \Gamma_{l}^{(j)}\right\|_{L^{2}\left(\widehat{\mathscr{J}}_{\delta}^{(j)}\right)} \\
& \leq C \sqrt{1+\frac{1}{\sqrt{\delta}}}\left\|\Gamma_{l}^{(j)}\right\|_{\mathrm{BL}} .
\end{aligned}
$$


The first term can then be bounded as follows

$$
\delta^{l} \delta^{3 / 2}\left\|\Gamma_{l}^{(j)}\right\|_{L^{2}\left(\widehat{\mathscr{I}_{\delta}^{(j)}}\right)} \leq C \delta^{l+3 / 2-1 / 4}=C \delta^{l+5 / 4}
$$

It remains to get an upper bound for $\mathrm{U}_{l, l}$. It is defined by means of (11) and depends at most on the outer coefficients of order $\leq l$. Formula (17) gives that $u_{k}(x)$ is a meromorphic function with poles of order $\leq k$ at the $c_{j}$. As a result, the matching functions can be bounded as follows

$$
\left|\mathrm{U}_{l, l}^{(j)}(X)\right| \leq C|X|^{l}, \forall X \in{\widehat{\mathscr{O}^{(j)}}}^{c}
$$

yielding

$$
\begin{aligned}
\delta^{l+3 / 2}\left\|\mathrm{U}_{l, l}^{(j)}\right\|_{L^{2}\left(\widehat{\mathscr{I}}_{\delta}^{(j)}\right)} & \leq C \delta^{l+3 / 2}\left(\frac{1}{\sqrt{\delta}}\right)^{l+3 / 2} \\
& \leq C \delta^{l / 2+3 / 4} .
\end{aligned}
$$

Using the previous results for $l=m-1$ and $l=m$, we come to the bound announced in the statement of the lemma.

Matching errors of the first kind are dealt with in the following lemma.

Lemma 9 As in above lemma, we have

$$
\sum_{j=1}^{N}\left\|\Delta \chi_{\delta}^{(j)}\left(\sum_{k=0}^{m} \delta^{k}\left(\Pi_{k}^{(j)}-u_{k}\right)\right)\right\|_{L^{2}\left(\mathscr{M}_{\delta}^{(j)}\right)} \leq C \delta^{m / 2+1 / 4}
$$

Proof The chain rule directly yields

$$
\begin{aligned}
\Delta \chi_{\delta}^{(j)}(x) & =\sum_{i=1}^{3} \partial_{x_{i}}^{2} \chi\left(\frac{\left|x-c_{j}\right|}{\sqrt{\delta}}\right)=\frac{1}{\sqrt{\delta}} \sum_{i=1}^{3} \partial_{x_{i}}\left(\frac{x_{i}-\left(c_{j}\right)_{i}}{\left|x-c_{j}\right|} \chi^{\prime}\left(\frac{\left|x-c_{j}\right|}{\sqrt{\delta}}\right)\right) \\
& =\frac{1}{\delta} \chi^{\prime \prime}\left(\frac{\left|x-c_{j}\right|}{\sqrt{\delta}}\right)+\frac{2}{\sqrt{\delta}} \frac{1}{\left|x-c_{j}\right|} \chi^{\prime}\left(\frac{\left|x-c_{j}\right|}{\sqrt{\delta}}\right) .
\end{aligned}
$$

Therefore, since $\sqrt{\delta} /\left|x-c_{j}\right|<1$ for $x \in \mathscr{M}_{\delta}^{(j)}$, we can write

$$
\left\|\Delta \chi_{\delta}^{(j)}\left(\sum_{k=0}^{m} \delta^{k}\left(\Pi_{k}^{(j)}-u_{k}\right)\right)\right\|_{L^{2}\left(\mathscr{M}_{\delta}^{(j)}\right)} \leq \frac{C}{\delta}\left\|\sum_{k=0}^{m} \delta^{k}\left(\Pi_{k}^{(j)}-u_{k}\right)\right\|_{L^{2}\left(\mathscr{M}_{\delta}^{(j)}\right)},
$$


with a constant $C$ depending only on $\chi$. We now use matching conditions (12) to bound the above terms. Introducing the matching functions as follows

$$
\begin{aligned}
\left\|\sum_{k=0}^{m} \delta^{k}\left(\Pi_{k}^{(j)}-u_{k}\right)\right\|_{L^{2}\left(\mathscr{M}_{\delta}^{(j)}\right)} \leq & \left\|\sum_{k=0}^{m} \delta^{k}\left(\Pi_{k}^{(j)}-\mathrm{U}_{m, k}^{(j)}\right)\right\|_{L^{2}\left(\mathscr{M}_{\delta}^{(j)}\right)} \\
& +\left\|\sum_{k=0}^{m} \delta^{k}\left(u_{k}-\mathrm{U}_{m, k}^{(j)}\right)\right\|_{L^{2}\left(\mathscr{M}_{\delta}^{(j)}\right)},
\end{aligned}
$$

expressing them with respect to the fast variable $X=\left(x-c_{j}\right) / \delta$, and passing to spherical variables, we get

$$
\begin{aligned}
\| \sum_{k=0}^{m} \delta^{k} & \left(\Pi_{k}^{(j)}-\mathrm{U}_{m, k}^{(j)}\right) \|_{L^{2}\left(\mathscr{M}_{\delta}^{(j)}\right)}^{2}= \\
& \int_{\frac{1}{\sqrt{\delta}}}^{\frac{2}{\sqrt{\delta}}} \int_{\mathscr{S}^{2}}\left|\sum_{k=0}^{m} \delta^{k}\left(\Pi_{k}^{(j)}(R \Theta)-\mathrm{U}_{m, k}^{(j)}(R \Theta)\right)\right|^{2} \delta^{3} R^{2} d \Theta d R
\end{aligned}
$$

where $\mathscr{S}^{2}$ is the unit sphere of $\mathbb{R}^{3}$. Making use of the sharp asymptotic behavior (22)

$$
\mathrm{U}_{m, k}^{(j)}-\Pi_{k}^{(j)}=\underset{R \rightarrow+\infty}{O}\left(\frac{1}{R^{m-k+1}}\right)
$$

we come to

$$
\begin{aligned}
\left\|\sum_{k=0}^{m} \delta^{k}\left(\Pi_{k}^{(j)}-\mathrm{U}_{m, k}^{(j)}\right)\right\|_{L^{2}\left(\mathscr{M}_{\delta}^{(j)}\right)} & \leq C \sum_{k=0}^{m} \delta^{k+\frac{3}{2}}\left(\int_{\frac{1}{\sqrt{\delta}}}^{\frac{2}{\sqrt{\delta}}} \frac{1}{R^{2 m-2 k+2}} R^{2} d R\right)^{\frac{1}{2}} \\
& \leq C \sum_{k=0}^{m} \delta^{k+\frac{3}{2}} \delta^{(2 m-2 k-1) / 4} \leq C \delta^{\frac{m}{2}+\frac{5}{4}}
\end{aligned}
$$

In the same way, we have

$$
\begin{aligned}
\left\|\sum_{k=0}^{m} \delta^{k}\left(u_{k}-\mathrm{U}_{m, k}^{(j)}\right)\right\|_{L^{2}\left(\mathscr{M}_{\delta, 1}^{(j)}\right)} & \leq C \delta^{\frac{3}{2}+m}\left(\int_{\frac{1}{\sqrt{\delta}}}^{\frac{2}{\sqrt{\delta}}} R^{2} d R\right)^{\frac{1}{2}} \\
& \leq C \delta^{m+\frac{3}{2}} \delta^{-\frac{3}{4}}=C \delta^{m+\frac{3}{4}}
\end{aligned}
$$

Gathering the previous estimates, we arrive to

$$
\begin{aligned}
& \left\|\Delta \chi_{\delta}^{(j)} \sum_{k=0}^{m} \delta^{k}\left(\Pi_{k}^{(j)}-u_{k}\right)\right\|_{L^{2}\left(\mathscr{M}_{\delta}^{(j)}\right)} \leq C\left(\delta^{\frac{m}{2}+\frac{1}{4}}+\delta^{m+\frac{3}{4}}\right) \\
& \leq C \delta^{\frac{m}{2}+\frac{1}{4}} .
\end{aligned}
$$


The last matching error term is dealt with in the following lemma.

Lemma 10 There exists a constant $C>0$ independent of $\delta$ such that:

$$
\sum_{j=1}^{N}\left\|\sum_{k=0}^{m} \delta^{k} \nabla \chi_{\delta}^{(j)} \cdot \nabla\left(\Pi_{k}^{(j)}-u_{k}\right)\right\|_{L^{2}\left(\mathscr{M}_{\delta}^{(j)}\right)} \leq C \delta^{m / 2+7 / 4}
$$

Proof As above, we have

$$
\nabla \chi_{\delta}^{(j)}(x)=\frac{1}{\sqrt{\delta}} \chi^{\prime}\left(\frac{\left|x-c_{j}\right|}{\sqrt{\delta}}\right) \frac{x-c_{j}}{\left|x-c_{j}\right|},
$$

Passing to the fast variable, we can write

$$
\begin{aligned}
\nabla \chi_{\delta}^{(j)}(x) & \nabla_{x}\left(\Pi_{k}^{(j)}\left(\frac{x-c_{j}}{\delta}\right)-u_{k}(x)\right)= \\
& \frac{1}{\delta \sqrt{\delta}} \chi^{\prime}\left(\frac{\left|x-c_{j}\right|}{\sqrt{\delta}}\right) \partial_{R}\left(\Pi_{k}^{(j)}(R \Theta)-u_{k}\left(c_{j}+\delta R \Theta\right)\right) .
\end{aligned}
$$

We then obtain

$$
\begin{aligned}
\| \sum_{k=0}^{m} \delta^{k} \nabla_{x} \chi_{\delta}^{(j)} & \cdot \nabla_{x}\left(\Pi_{k}^{(j)}-u_{k}\right) \|_{L^{2}\left(\mathscr{M}_{\delta}^{(j)}\right)} \\
\leq & \frac{C}{\delta^{3 / 2}}\left\|\sum_{k=0}^{m} \delta^{k} \partial_{R}\left(\Pi_{k}^{(j)}-u_{k}\right)\right\|_{L^{2}\left(\mathscr{M}_{\delta}^{(j)}\right)} \\
\leq & \frac{C}{\delta^{3 / 2}}\left\|\sum_{k=0}^{m} \delta^{k} \partial_{R}\left(\Pi_{k}^{(j)}-\mathrm{U}_{m, k}^{(j)}\right)\right\|_{L^{2}\left(\mathscr{M}_{\delta}^{(j)}\right)} \\
& +\frac{C}{\delta^{3 / 2}}\left\|\sum_{k=0}^{m} \delta^{k} \partial_{R}\left(u_{k}-\mathrm{U}_{m, k}^{(j)}\right)\right\|_{L^{2}\left(\mathscr{M}_{\delta}^{(j)}\right)}
\end{aligned}
$$

with a positive constant $C$ independent of $\delta$. We now need to get bounds on the derivatives of the matching rules. Chain rule $\partial_{R}=\delta \partial_{\left|x-c_{j}\right|}$ and the fact that $c_{j}$ is a pole for the meromorphic function $\sum_{k=0}^{m} \delta^{l} u_{k}(x)-\sum_{k=0}^{m} \delta^{l} \mathrm{U}_{m, l}^{(j)}\left(\left(x-c_{j}\right) / \delta\right)$ yield

$$
\partial_{R}\left(\sum_{k=0}^{m} \delta^{l} u_{k}\left(c_{j}+\delta R \Theta\right)-\sum_{k=0}^{m} \delta^{l} \mathrm{U}_{m, l}^{(j)}(R \Theta)\right)=\underset{\delta \rightarrow 0}{O}\left(\delta^{m+1}\right) .
$$

We then obtain:

$$
\left\|\sum_{k=0}^{m} \delta^{k} \partial_{R}\left(u_{k}-\mathrm{U}_{m, k}^{(j)}\right)\right\|_{L^{2}\left(\mathscr{M}_{\delta}^{(j)}\right)} \leq C \delta^{\frac{3}{2}+m+1}\left(\int_{\frac{1}{\sqrt{\delta}}}^{\frac{2}{\sqrt{\delta}}} R^{2} d R\right)^{\frac{1}{2}} \leq C \delta^{m+\frac{7}{4}}
$$

To get bounds for the derivatives of the matching rules, we use lemma 3 which shows that the $\mathrm{U}_{m, k}^{(j)}-\Pi_{k}^{(j)}$ satisfy the recursive Laplace equations with conditions at infinity given by the matching rules (12). In view of (21), we can then argue that

$$
\partial_{R}\left(\mathrm{U}_{m, k}^{(j)}-\Pi_{k}^{(j)}\right)=\underset{R \rightarrow+\infty}{O}\left(\frac{1}{R^{m-k+2}}\right) .
$$


This directly leads to

$$
\begin{aligned}
& \| \sum_{k=0}^{m} \delta^{k} \partial_{R}(\left.\Pi_{k}^{(j)}-\mathrm{U}_{m, k}^{(j)}\right) \|_{L^{2}\left(\mathscr{M}_{\delta}^{(j)}\right)} \\
& \leq C \sum_{k=0}^{m} \delta^{k+\frac{3}{2}}\left(\int_{\frac{1}{\sqrt{\delta}}}^{\frac{2}{\sqrt{\delta}}}\left|\frac{1}{R^{m-k+2}}\right|^{2} R^{2} d R\right)^{\frac{1}{2}} \\
& \leq C \sum_{k=0}^{m} \delta^{k+\frac{3}{2}}\left(\delta^{2 m-2 k+1}\right)^{\frac{1}{4}} \leq C \delta^{\frac{m}{2}+\frac{7}{4}}
\end{aligned}
$$

Gathering the previous estimates, we get

$$
\left\|\sum_{k=0}^{m} \delta^{k} \nabla \chi_{\delta}^{(j)} \cdot \nabla\left(\Pi_{k}^{(j)}-u_{k}\right)\right\|_{L^{2}\left(\mathscr{M}_{\delta}^{(j)}\right)} \leq C \delta^{\frac{m}{2}+\frac{7}{4}}
$$

with $C$ a constant independent of $\delta$.

\subsection{Final and optimal error estimates}

From the stability result (36) and the above bounds on the residual, we directly obtain the following bound for the uniformly valid approximation.

Theorem 4 Let $u_{m, \delta}$ be the above uniformly valid approximation of the solution $u_{\delta}$ to scattering problem (3) with any of the above conditions (4), (5), or (6) on the small objects. The following bound holds true

$$
\left\|u_{\delta}-u_{m, \delta}\right\|_{H^{1}\left(\Upsilon_{\delta}\right)} \leq C \delta^{\frac{1}{4}+\frac{m}{2}}
$$

with a positive constant $C$ independent of $\delta$.

The previous theorem gives a theoretical justification for the above inner and outer expansions. The obtained error estimate is of course not optimal. This is due as usual in this kind of issues to the two-scale character of the solution. An optimal error estimate can however be retrieved outside suitable fixed neighborhoods of the small obstacles.

\section{Theorem 5 Let}

$$
\mathscr{F}_{\rho}=\Upsilon \backslash \overline{\cup_{j=1}^{N} \mathscr{B}_{c_{j}}^{\rho}}
$$


where $\rho$ is fixed sufficiently small positive number. There exist $\delta_{0}>0$ and a positive constant $C_{\rho}$ such that for $0<\delta \leq \delta_{0}$ the following optimal error estimate holds true

$$
\left\|u_{\delta}-\sum_{k=0}^{m} \delta^{k} u_{k}\right\|_{H^{1}\left(\mathscr{F}_{\rho}\right)} \leq C_{\rho} \delta^{m+1} .
$$

Proof Clearly, for $\rho$ sufficiently small the balls $\mathscr{B}_{c j}^{\rho}$ do not intersect. In the same way, for $0<\delta \leq \delta_{0}$ with $\delta_{0}$ sufficiently small $\left.u_{m, \delta}\right|_{\mathscr{F}_{\rho}}=\sum_{k=0}^{m} \delta^{k} u_{k}$. Now for an integer $p$ such that $p \geq m$ and $p / 2+1 / 4 \geq m+1$, the error estimate on the uniformly valid approximation yields

$$
\left\|u_{\delta}-u_{p, \delta}\right\|_{H^{1}\left(r_{\delta}\right)} \leq C \delta^{\frac{1}{4}+\frac{p}{2}} \leq C \delta^{m+1} .
$$

Using the triangular inequality, we readily obtain

$$
\left\|u_{\delta}-u_{m, \delta}\right\|_{H^{1}(\mathscr{F} \rho)} \leq\left\|u_{\delta}-u_{p, \delta}\right\|_{H^{1}\left(r_{\delta}\right)}+\sum_{l=m+1}^{p} \delta^{l}\left\|u_{l}\right\|_{H^{1}\left(\mathscr{F}_{\rho}\right)}
$$

thus completing the proof of the theorem.

We have thus completed the proof that the effect of each of the small obstacles can be approximated by a suitable multipole, the order of which increasing with the accuracy which is being sought. We will see at the end of this paper to what kind of other scatterers can or cannot be drawn the same conclusion.We are going now to show how this analysis can be used to obtain a mathematical justification of Foldy's usual reduced model in which each small obstacle is replaced by an isotropic point scatterer and more importantly how this enables us to improve it.

\section{Foldy's model and its improvement}

After introducing two boundary-value problems involved in the determination of the inner expansion up to order 2 , we give a characterization of the asymptotic behavior at infinity of their solution. This will enable us in a first step to establish an optimal bound for the error induced by Foldy's model and next to improve it. 
5.1 Two fundamental exterior boundary-value problems

In this part, we give a characterization of the asymptotic behavior at infinity of two boundary-value problems involved in the explicit determination of the inner asymptotic expansion of $u_{\delta}$ up to order 2 . The first of these has been already introduced in (25).

\subsubsection{The exterior boundary-value problems}

The second exterior boundary-value problem is stated as follows

$$
\left\{\begin{array}{l}
\Delta \Lambda^{(j)}=0 \text { in } \widehat{\mathscr{O}}^{c j}{ }^{c}, \\
B^{(j)} \Lambda^{(j)}=0 \text { on } \partial \widehat{\mathscr{O}^{(j)}}, \\
\Lambda^{(j)}(X)=X+\underset{|X| \rightarrow \infty}{o}(1) .
\end{array}\right.
$$

It is worth underlying that problem (40) actually consists of three uncoupled problems each of them posed for one component of $\Lambda^{(j)}$.

As for $\Phi^{(j)}$, we seek a solution to (40) in the form $\Lambda^{(j)}=X+\Lambda_{\mathrm{BL}}^{(j)}$ with $\Lambda_{\mathrm{BL}}^{(j)}$ in the usual Beppo-Levi space (cf., e.g., $[24,20]$ ). we readily get that this problem too has one and only one solution. Clearly, the same conclusion can be drawn for either a Dirichlet or a Neumann condition on $\partial \widehat{\mathscr{O}^{(j)}}$.

\subsubsection{Asymptotic expansion at infinity}

Using the well-posedness of the interior Dirichlet problem for the Laplace equation, we easily get that the above scalar $\Phi^{(j)}$ and vectorial functions $\Lambda^{(j)}$ admit the following integral representations in terms of a single-layer potential

$$
\left\{\begin{array}{l}
\Phi^{(j)}(X)=1+\int_{\partial \widehat{\mathscr{O}}^{(j)}} \frac{1}{|X-Y|} \varphi^{(j)}(Y) d s_{Y}, X \in \widehat{\mathscr{O}}^{c}{ }^{c} \\
\Lambda^{(j)}(X)=X+\int_{\partial \widehat{\mathscr{O}}^{(j)}} \frac{1}{|X-Y|} \lambda(Y) d s_{Y}, X \in \widehat{\mathscr{O}}^{c}{ }^{c}
\end{array}\right.
$$

It is well-known that the Green kernel 1/ $|X-Y|$ can be expanded for $|Y|<|X|$ using the generating function for Legendre polynomials $\mathrm{P}_{n}(t)$

$$
\frac{1}{\sqrt{1-2 x t+t^{2}}}=\sum_{n=0}^{\infty} \mathrm{P}_{n}(x) t^{n} \text { for }|t|<\min \left(\left|x \pm \sqrt{x^{2}-1}\right|\right)
$$


as an entire function of $|Y| /|X|$ (see, e.g., [30, Formula (2), p. 215])

$$
\frac{1}{|X-Y|}=\sum_{n=0}^{\infty} \mathrm{P}_{n}\left(\frac{X}{|X|} \cdot \frac{Y}{|Y|}\right) \frac{|Y|^{n}}{|X|^{n+1}} .
$$

As a result, the above two functions can be expanded as follows for $|X| \rightarrow \infty$

$$
\left\{\begin{array}{l}
\Phi^{(j)}(X)=1+\sum_{n=0}^{\infty} \frac{\sigma_{n}^{(j)}(X /|X|)}{|X|^{n+1}} \\
\Lambda^{(j)}(X)=X+\sum_{n=0}^{\infty} \frac{S_{n}^{(j)}(X /|X|)}{|X|^{n+1}}
\end{array}\right.
$$

with

$$
\left\{\begin{array}{l}
\sigma_{n}^{(j)}(X /|X|)=\int_{\partial \widehat{\mathscr{O}}^{(j)}} \mathrm{P}_{n}\left(\frac{X}{|X|} \cdot \frac{Y}{|Y|}\right)|Y|^{n} \varphi^{(j)}(Y) d s_{Y}, \\
S_{n}^{(j)}(X /|X|)=\int_{\partial \widehat{\mathscr{O}}^{(j)}} \mathrm{P}_{n}\left(\frac{X}{|X|} \cdot \frac{Y}{|Y|}\right)|Y|^{n} \lambda^{(j)}(Y) d s_{Y} .
\end{array}\right.
$$

Actually, since $\mathrm{P}_{0}(x)=1$ and $\mathrm{P}_{1}(x)=x, \sigma_{0}^{(j)}, \sigma_{1}^{(j)}$, and $S_{0}^{(j)}$ are expressed in terms of the respective moments of $\varphi^{(j)}$ and $\lambda^{(j)}$ of order 0 or 1

$$
\left\{\begin{array}{l}
\sigma_{0}^{(j)}=\int_{\partial \widehat{\mathscr{O}}^{(j)}} \varphi^{(j)}(Y) d s_{Y} \\
\Sigma_{1}^{(j)}=\int_{\partial \widehat{\widehat{O}^{(j)}}} Y \varphi^{(j)}(Y) d s_{Y} \\
\sigma_{1}^{(j)}(X /|X|)=\Sigma_{1}^{(j)} \cdot X /|X|
\end{array}\right.
$$

In the same way, $S_{0}^{(j)}$ is constant and is given by

$$
S_{0}^{(j)}=\int_{\partial \widehat{\mathscr{O}^{(j)}}} \lambda^{(j)}(Y) d s_{Y}
$$

Remark 6 It should be mentionned that, as regard with the Neumann condition for $\Phi^{(j)}$, since $\Phi_{\mathrm{BL}}^{(j)}=0$ (see Remark 4 above), any of the coefficients $\sigma_{n}^{(j)}$ is zero.

\subsubsection{Some properties of the coefficients of the asymptotic expansion at infinity}

We begin with a kind of reciprocity theorem.

Lemma 11 Recall that $\mathscr{S}^{2}$ denotes the unit sphere of $\mathbb{R}^{3}$. The above coefficients $\sigma_{1}^{(j)}$ and $S_{0}^{(j)}$ satisfy

$$
S_{0}^{(j)}=\int_{\mathscr{S}^{2}} 3 \sigma_{1}^{(j)}(\widehat{X}) \widehat{X} d s_{\widehat{X}}
$$


Proof Let us denote by $\mathscr{O}_{R}^{(j)}={\widehat{\mathcal{O}^{(j)}}}^{c} \cap \mathscr{B}_{R}$ with $\mathscr{B}_{R}=\left\{x \in \mathbb{R}^{3} ;|x|<R\right\}$. Green formula then yields

$$
\begin{gathered}
\int_{\mathscr{O}_{R}^{(j)}}\left(\Phi^{(j)} \Delta \Lambda^{(j)}-\Lambda^{(j)} \Delta \Phi^{(j)}\right) d x=-\int_{\partial \widehat{\partial(j)}}\left(\Phi^{(j)} \partial_{\widehat{n}_{j}} \Lambda^{(j)}-\Lambda^{(j)} \partial_{\widehat{\mathbf{n}}_{j}} \Phi^{(j)}\right) d s \\
+R^{2} \int_{\widehat{S}}\left(1+\frac{\sigma_{0}^{(j)}}{R}+\frac{\sigma_{1}^{(j)}(\widehat{X})}{R^{2}}+O\left(\frac{1}{R^{3}}\right)\right)\left(\widehat{X}-\frac{S_{0}^{(j)}}{R^{2}}+O\left(\frac{1}{R^{3}}\right)\right) d s \\
-R^{2} \int_{\mathscr{S}^{2}}\left(-\frac{\sigma_{0}^{(j)}}{R^{2}}-\frac{2 \sigma_{1}^{(j)}(\widehat{X})}{R^{3}}+O\left(\frac{1}{R^{4}}\right)\right)\left(R \widehat{X}+O\left(\frac{1}{R}\right)\right) d s
\end{gathered}
$$

The boundary condition in problems $(25)$ and (40) give that the integral on $\partial \widehat{\mathscr{O}^{(j)}}$ is zero. By symmetry we also have

$$
\int_{\mathscr{S}^{2}} \widehat{X} d s=0 .
$$

As a result, we get from the above relation

$$
\int_{\mathscr{S}^{2}} 3 \sigma_{1}^{(j)}(\widehat{X}) \widehat{X} d s_{\widehat{X}}-S_{0}^{(j)}+O\left(\frac{1}{R}\right)=0
$$

readily yielding (48) by passing to the limit as $R \rightarrow \infty$.

Remark 7 Note that the above lemma gives in particular that $S_{0}^{(j)}=0$ when dealing with a Neumann condition.

We are now going to establish that we can always ensure that $\sigma_{1}^{(j)}=0$ by a change of variables of the form

$$
X=X^{\prime}+X_{0}
$$

obtained by suitably translating the origin. This is precisely the way, which was previously announced, for adequately fixing the centers of phases $c_{j}$ in Foldy's model to improve its accuracy. We first prove the following intermediary lemma.

Lemma 12 The following formula holds true

$$
\mathfrak{I} \sigma_{0}^{(j)}=-\frac{\mathfrak{I} v_{j}}{4 \pi} \int_{\partial \widehat{\mathscr{O}}^{(j)}}\left|\Phi^{(j)}\right|^{2} d s
$$

and thus $\sigma_{0}^{(j)}$ satisfies

$$
\sigma_{0}^{(j)} \neq 0
$$

when dealing with the impedance boundary condition. 
Proof We proceed as in the proof of the above lemma and use Green's formula to write

$$
\begin{aligned}
\int_{\mathscr{O}_{R}^{(j)}}\left(\overline{\Phi^{(j)}} \Delta \Phi^{(j)}+\left|\nabla \Phi^{(j)}\right|^{2}\right) d x & =-\int_{\partial \widehat{\widehat{O}^{(j)}}} \overline{\Phi^{(j)}} \partial_{\widehat{\mathbf{n}_{j}}} \Phi^{(j)} d s \\
& +R^{2} \int_{\mathscr{S}^{2}}\left(1+O\left(\frac{1}{R}\right)\right)\left(-\frac{\sigma_{0}^{(j)}}{R^{2}}+O\left(\frac{1}{R^{3}}\right)\right) d s .
\end{aligned}
$$

Expanding and passing to the limit as $R \rightarrow \infty$, we directly get

$$
\int_{\widehat{\widehat{O}^{(j)}}}\left|\nabla \Phi^{(j)}\right|^{2} d x+\int_{\partial \widehat{\widehat{O}^{(j)}}} \overline{\Phi^{(j)}} \partial_{\widehat{\mathbf{n}}_{j}} \Phi^{(j)} d s+4 \pi \sigma_{0}^{(j)}=0
$$

which directly yields formula (50). The rest of the proof is a consequence of condition (16) and the fact that Cauchy data $\left.\Phi^{(j)}\right|_{\partial \widehat{\mathscr{O}(j)}}$ and $\left.\partial_{\widehat{\mathbf{n}}_{j}} \Phi^{(j)}\right|_{\partial \widehat{\mathscr{C}(j)}}$ of $\Phi^{(j)}$ cannot be zero simultaneously.

Remark 8 We have already pointed out that $\sigma_{0}^{(j)}=0$ for the Neumann condition. The above property $\sigma_{0}^{(j)} \neq 0$ holds for the Dirichlet condition also by using (52).

Let us now define $\Psi^{(j)}$ the function obtained from $\Phi^{(j)}$ by the above variable change (49)

$$
\Psi^{(j)}(Y)=\Phi^{(j)}\left(Y+X_{0}\right) .
$$

This function satisfies the same boundary-value problem than $\Phi^{(j)}$. Expanding $1 /|X|$ for sufficiently large $|Y|$ as follows

$$
\frac{1}{\left|Y+X_{0}\right|}=\frac{1}{|Y|} \sum_{n=0}^{\infty}(-1)^{n} \mathrm{P}_{n}\left(\frac{Y}{|Y|} \cdot \frac{X_{0}}{\left|X_{0}\right|}\right) \frac{\left|X_{0}\right|^{n}}{|Y|^{n}}
$$

we get the following asymptotic expansion for $\Psi^{(j)}$ as $|Y| \rightarrow \infty$

$$
\Psi^{(j)}(Y)=1+\frac{\sigma_{0}^{(j)}}{|Y|}+\frac{1}{|Y|^{2}}\left(\Sigma_{1}^{(j)}-\sigma_{0}^{(j)} X_{0}\right) \cdot \frac{Y}{|Y|}+O\left(\frac{1}{|Y|^{3}}\right) .
$$

We have thus almost proved the following theorem.

Theorem 6 The two first coefficients $\sigma_{0}^{(j)}$ and $\Sigma_{1}^{(j)}$ of the asymptotic expansion of $\Phi^{(j)}$ for $|X| \rightarrow \infty$ have to be modified according to the variable change (49) as follows

$$
\sigma_{0, X_{0}}^{(j)}=\sigma_{0}^{(j)}, \quad \Sigma_{1, X_{0}}^{(j)}=\Sigma_{1}^{(j)}-\sigma_{0}^{(j)} X_{0}
$$


Therefore, by possibly making the above variable change, we can always assume that

$$
\Sigma_{1}^{(j)}=0
$$

and also, from (48), that

$$
S_{0}^{(j)}=0
$$

Proof For boundary condition of problem (25) and for the case of a Dirichlet boundary condition, we obtain (56) by passing to the variable $Y$ with

$$
X_{0}=\frac{1}{\sigma_{0}^{(j)}} \Sigma_{1}^{(j)} .
$$

The case of a Neumann condition does not require any change: both $\sigma_{0}^{(j)}, \Sigma_{1}^{(j)}$, and $S_{0}^{(j)}$ are then zero.

\subsection{The usual Foldy model}

We first determine the first-order outer asymptotic expansion of $u_{\delta}$ explicitely and show how Foldy's usual model can then be derived and error estimates established.

\subsubsection{Explicit determination of the first-order outer asymptotic expansion}

We follow the general procedure already given above. Both the outer and inner zeroth-order asymptotic expansions have been determined. We just recall that $u_{0}$ is obtained by just disgarding the effects of the small obstacles. The inner expansion is obtained for each small object by

$$
\Pi_{0}^{(j)}(X)=u_{0}\left(c_{j}\right) \Phi^{(j)}(X)
$$

where $\Phi^{(j)}$ is one of the two functions introduced above.

The matching rules give that the first-order expansion contains at most monopole sources located at the $c_{j}$

$$
u_{1}(x)=\sum_{\ell=1}^{N} h_{0}^{(1)}\left(\kappa\left|x-c_{\ell}\right|\right) Y_{0}^{(j)}
$$

where $h_{0}^{(1)}$ is the first kind Hankel function of order 0 and $Y_{0}^{(j)}$ is a yet undetermined spherical harmonic function of order zero, really a constant. 
To determine $Y_{0}^{(j)}$, we have first to define the matching function $\mathrm{U}_{0,1}^{(j)}$ from the following expansion

$$
\begin{aligned}
u_{1 \delta}\left(c_{j}+\delta X\right) & =u_{0}\left(c_{j}+\delta X\right)+\delta u_{1}\left(c_{j}+\delta X\right) \\
& =\mathrm{U}_{1,0}^{(j)}(X)+\underset{\delta \rightarrow 0}{o}(1) .
\end{aligned}
$$

Since

$$
h_{0}^{(1)}(z)=\frac{e^{i z}}{i z}=\frac{1}{i z}+\frac{i z}{2}+\underset{z \rightarrow 0}{o}(z)
$$

we readily get

$$
\mathrm{U}_{1,0}^{(j)}(X)=u_{0}\left(c_{j}\right)+\frac{Y_{0}^{(j)}}{i \kappa|X|}
$$

Matching rules

$$
\Pi_{0}^{(j)}(X)-\mathrm{U}_{1,0}^{(j)}(X)=\underset{|X| \rightarrow \infty}{o}\left(\frac{1}{|X|}\right)
$$

given above in (12) then yield

$$
Y_{0}^{(j)}=i \kappa \sigma_{0}^{(j)} u_{0}\left(c_{j}\right)
$$

First-order outer coefficient $u_{1}$ is hence obtained as the field created by the following monopoles placed at the centers of phase $c_{j}$

$$
u_{1}(x)=i \kappa \sum_{j=1}^{N} \sigma_{0}^{(j)} u_{0}\left(c_{j}\right) h_{0}^{(1)}\left(\kappa\left|x-c_{j}\right|\right) .
$$

\subsubsection{Derivation of the usual Foldy model}

In view of the above calculation, up to the first-order terms in $\delta$, the total field resulting from the scattering of an incident field $w$ which would be present in the proximity of $c_{j}$ without the small scatterer placed there, is given by

$$
w(x)+i \kappa \delta \sigma_{0}^{(j)} w\left(c_{j}\right) h_{0}^{(1)}\left(\kappa\left|x-c_{j}\right|\right)
$$

This is the crucial assumption of Foldy's isotropic model. The scattering problem related to this model is set by looking to an approximation of the total field as the superposition of the incident field $u_{\text {inc }}$ and $N$ unknown monopoles

$$
u_{\delta}^{\mathrm{F}}(x)=u_{\text {inc }}(x)+\sum_{\ell=1}^{N} A^{(\ell)} h_{0}^{(1)}\left(\kappa\left|x-c_{\ell}\right|\right) .
$$


(see, e.g, $[14,22])$ whose strengths can be determined by solving the following linear system

$$
\left\{\begin{array}{r}
A^{(j)}=i \kappa \delta \sigma_{0}^{(j)}\left(u_{\mathrm{inc}}\left(c_{j}\right)+\sum_{\substack{1 \leq \ell \leq N \\
\ell \neq j}} A^{(\ell)} h_{0}^{(1)}\left(\kappa\left|c_{j}-c_{\ell}\right|\right)\right) \\
(1 \leq j \leq N) .
\end{array}\right.
$$

Clearly, if $A$ denotes the column-wise vector collecting the $A^{(j)}$, the above system can be put in the following compact form

$$
A-\delta \Xi A=\delta B
$$

where $\Xi$ is the $N \times N$ matrix whose coefficients are $\Xi_{j l}=i \kappa \sigma_{0}^{(j)} h_{0}^{(1)}\left(\kappa\left|c_{j}-c_{\ell}\right|\right)$ for $j \neq l$ and $\Xi_{l l}=0$, and $B_{j}=i \kappa \sigma_{0}^{(j)} u_{\text {inc }}\left(c_{j}\right)$. System (68) is set in term of a Neumann matrix obtained as a pertrurbation of the identity matrix as $\delta \rightarrow 0$. The proof of the following lemma can be readily obtained from the explicit entire series expansion of the inverse of $I-\delta \Xi$

$$
(I-\delta \Xi)^{-1}=I+\delta \Xi+\delta^{2} \Xi^{2}+\cdots
$$

Lemma 13 System (68) can be uniquely solved in a stable way as $\delta \rightarrow 0$. Moreover, its solution admits the following expansion

$$
A=A_{0}+\delta A_{1}+\cdots+\delta^{n} A_{n}+\underset{\delta \rightarrow \infty}{o}\left(\delta^{n}\right)
$$

whose coefficients are recursively defined by

$$
A_{0}=0, A_{1}=B, A_{n+1}=\Xi A_{n}(n=1,2, \ldots)
$$

Hence,

$$
A_{1}^{(j)}=i \kappa \sigma_{0}^{(j)} u_{0}\left(c_{j}\right)
$$

In other words, the 1 st-order expansion of $u_{\delta}^{\mathrm{F}}$ in powers of $\delta$ coincides with the outer expansion of $u_{\delta}$. As a result, we get the following theorem containing the justification of Foldy's isotropic model from above theorem that establishes optimal error bounds for the outer approximation of $u_{\delta}$ in section 5 
Theorem 7 Let $u_{\delta}$ be the total field of scattering problem (3) with impedance (4) or sound-soft boundary condition on the small objects. Let $u_{\delta}^{F}$ be the solution (66) obtained from the above Foldy model. Then, there exist $\delta_{0}, \rho>0$ and a constant $C_{\rho}$ such that for $0<\delta \leq \delta_{0}$ the following optimal bound holds true

$$
\left\|u_{\delta}-u_{\delta}^{F}\right\|_{H^{1}(\mathscr{F} \rho)} \leq C_{\rho} \delta^{2}
$$

(see Theorem 5 for the definition of $\mathscr{F} p$ ).

Remark 9 Clearly, using the usual integral representation of the far field $a_{\delta}(\Theta)$ $\left(\Theta \in \widehat{\mathscr{S}_{3}}\right), \widehat{\mathscr{S}_{3}}$ being the unit ball of $\mathbb{R}^{3}$, as defined in (3) (see e.g. [24]), and defining in the same way the far field $a_{\delta}^{\mathrm{F}}(\Theta)$ corresponding to the field $u_{\delta}^{\mathrm{F}}$ provided by the Foldy model, we readily get the following estimate

$$
\left\|a_{\delta}-a_{\delta}^{\mathrm{F}}\right\|_{H^{s}\left(\widehat{\mathscr{S}_{3}}\right)} \leq C_{s} \delta^{2} \quad(s \in \mathbb{R})
$$

with a constant $C_{s}$ independent of $\delta$.

\subsection{The improved Foldy model}

We now explicitely expand both the exact solution $u_{\delta}$ of the multi-scattering problem and its approximation $u_{\delta}^{\mathrm{F}}$ through the usual Foldy-Lax model up to order 2. This will enable us to see that it is possible by adequately choosing the centers of phases $c_{j}$ and suitably writing this model to gain one further order of convergence.

\subsubsection{Explicit determination of the 2nd-order outer asymptotic expansion}

We first determine the 1st-order asymptotic expansion of $u_{\delta}$. Expanding

$$
\begin{aligned}
u_{1 \delta}\left(c_{j}+\delta X\right) & =u_{0}\left(c_{j}+\delta X\right)+ \\
& \delta\left(\begin{array}{l}
\sum_{\substack{\ell=1 \\
\ell \neq j}}^{N} i \kappa \sigma_{0}^{(\ell)} u_{0}\left(c_{\ell}\right) h_{0}^{(1)}\left(\kappa\left|c_{j}-c_{\ell}+\delta X\right|\right) \\
+i \kappa \sigma_{0}^{(j)} u_{0}\left(c_{j}\right) h_{0}^{(1)}(\kappa \delta|X|)
\end{array}\right) \\
& =\mathrm{U}_{1,0}^{(j)}(X)+\delta \mathrm{U}_{1,1}^{(j)}(X)+\underset{\delta \rightarrow 0}{o}(\delta),
\end{aligned}
$$


we get the matching function

$$
\mathrm{U}_{1,1}^{(j)}(X)=\nabla u_{0}\left(c_{j}\right) \cdot X+d^{(j)}
$$

with

$$
d^{(j)}=\sum_{\substack{\ell=1 \\ \ell \neq j}}^{N} i \kappa \sigma_{0}^{(\ell)} u_{0}\left(c_{\ell}\right) h_{0}^{(1)}\left(\kappa \delta\left|c_{j}-c_{\ell}\right|\right)+i \kappa \sigma_{0}^{(j)} u_{0}\left(c_{j}\right) .
$$

Above matching rules (12) then give

$$
\Pi_{1}^{(j)}(X)=\nabla u_{0}\left(c_{j}\right) \cdot \Lambda^{(j)}(X)+d^{(j)} \Phi^{(j)}(X)
$$

where $\Lambda^{(j)}$ is defined above as the solution to (40).

Matching rules once more compel $u_{2}$ to be in the following form

$$
u_{2}(x)=\sum_{\ell=1}^{N} h_{0}^{(1)}\left(\kappa\left|x-c_{\ell}\right|\right) Y_{0,2}^{(\ell)}+\sum_{\ell=1}^{N} h_{1}^{(1)}\left(\kappa\left|x-c_{\ell}\right|\right) Y_{1,2}^{(\ell)}
$$

where $Y_{k, 2}^{(\ell)}$ are undetermined spherical harmonics of degree $k(k=0,1)$ and $h_{1}^{(1)}(z)$ is the first kind spherical Hankel function of order 1 (cf., e.g., [15])

$$
h_{1}^{(2)}(z)=-\partial_{z} \frac{e^{i z}}{i z}=-\frac{e^{i z}}{z^{2}}(i+z) .
$$

Since

$$
h_{1}^{(2)}(z)=-\frac{i}{z^{2}}-\frac{i}{2}+\underset{z \rightarrow 0}{o}(1)
$$

then

$$
\begin{aligned}
u_{2 \delta}\left(c_{j}+\delta X\right) & =\left(u_{0}+\delta u_{1}+\delta^{2} u_{2}\right)\left(c_{j}+\delta X\right) \\
& =\mathrm{U}_{2,0}^{(j)}(X)+\delta \mathrm{U}_{2,1}^{(j)}(X)+\underset{\delta \rightarrow 0}{o}(\delta)
\end{aligned}
$$

so that

$$
\left\{\begin{array}{l}
\mathrm{U}_{2,0}^{(j)}(X)=\mathrm{U}_{1,0}^{(j)}(X)-\frac{i}{\kappa^{2}|X|^{2}} Y_{1,2}^{(j)} \\
\mathrm{U}_{2,1}^{(j)}(X)=\mathrm{U}_{1,1}^{(j)}(X)-\frac{i}{\kappa|X|} Y_{0,2}^{(j)}
\end{array}\right.
$$

Since

$$
\left\{\begin{array}{l}
\Pi_{0}^{(j)}(X)-\mathrm{U}_{2,0}^{(j)}(X)=\frac{1}{|X|^{2}} u_{0}\left(c_{j}\right) \sigma_{1}^{(j)} \cdot \frac{X}{|X|}+\frac{i}{\kappa^{2}|X|^{2}} Y_{1,2}^{(j)}+\underset{|X| \rightarrow \infty}{o}\left(\frac{1}{|X|^{2}}\right), \\
\Pi_{1}^{(j)}(X)-\mathrm{U}_{2,1}^{(j)}(X)=\frac{1}{|X|}\left(\nabla u_{0}\left(c_{j}\right) \cdot S_{0}^{(j)}+d^{(j)} \sigma_{0}^{(j)}\right)+\frac{i}{\kappa|X|} Y_{0,2}^{(j)}+\underset{|X| \rightarrow \infty}{o}\left(\frac{1}{|X|}\right),
\end{array}\right.
$$


matching rules then give

$$
\left\{\begin{array}{l}
Y_{1,2}^{(j)}=i \kappa^{2} u_{0}\left(c_{j}\right) \sigma_{1}^{(j)} \cdot \frac{X}{|X|} \\
Y_{0,2}^{(j)}=i \kappa\left(\sigma_{0}^{(j)} d^{(j)}+\nabla u_{0}\left(c_{j}\right) \cdot S_{0}^{(j)}\right) .
\end{array}\right.
$$

We have thus obtained that the second-order term in the outer expansion of $u_{\delta}$ reads

$$
\begin{aligned}
& u_{2}(x)=\sum_{j=1}^{N} i \kappa^{2} u_{0}\left(c_{j}\right) h_{1}^{(1)}\left(\kappa\left|x-c_{j}\right|\right) \sigma_{1}^{(j)} \cdot \frac{x-c_{j}}{\left|x-c_{j}\right|}+ \\
& \sum_{j=1}^{N} i \kappa \sigma_{0}^{(j)}\left(\begin{array}{c}
\sum_{\ell=1}^{N} i \kappa \sigma_{0}^{(\ell)} u_{0}\left(c_{\ell}\right) h_{0}^{(1)}\left(\kappa \delta\left|c_{j}-c_{\ell}\right|\right) \\
+i \kappa \sigma_{0}^{(j)} u_{0}\left(c_{j}\right)+\nabla u_{0}\left(c_{j}\right) \cdot S_{0}^{(j)}
\end{array}\right) h_{0}^{(1)}\left(\kappa\left|x-c_{j}\right|\right) .
\end{aligned}
$$

The second-order term of the asymptotic expansion of the solution to Foldy's model is obtained more easily from the above Neumann series expansion

$$
u_{2}^{\mathrm{F}}(x)=\sum_{j=1}^{N} i \kappa \sigma_{0}^{(j)}\left(\sum_{\substack{\ell=1 \\ \ell \neq j}}^{N} i \kappa \sigma_{0}^{(\ell)} u_{0}\left(c_{\ell}\right) h_{0}^{(1)}\left(\kappa \delta\left|c_{j}-c_{\ell}\right|\right)\right) h_{0}^{(1)}\left(\kappa\left|x-c_{j}\right|\right)
$$

\subsubsection{The improved Foldy model}

The 2nd-order outer coefficient (77) contains dipoles $i \kappa^{2} u_{0}\left(c_{j}\right) h_{1}^{(1)}\left(\kappa\left|x-c_{j}\right|\right) \Sigma_{1}^{(j)}$. $\left(x-c_{j}\right) /\left|x-c_{j}\right|$ which are not present in the same order one corresponding to the Foldy model. If we stop here with the derivation of the model, error bound (73) can no more be improved. Actually, a further order of convergence can be gained by suitably choosing the centers of phase $c_{j}$ instead of taking them rather intuitively. The correct procedure is the following.

- Take intuitively $c_{j}$ as previously indicated such that the $j$-th small body $\mathscr{O}_{\delta}^{(j)}$ is contained in the ball $\mathscr{B}_{c_{j}}^{\lambda \delta}$.

- Scale the variable $X=\left(x-c_{j}\right) / \delta$ and solve problem (25).

- Determine $\sigma_{0}^{(j)}$ and $\Sigma_{1}^{(j)}$ and in view of (55) and (58) correct the center of phase according to

$$
c_{j}^{\text {corr }}=c_{j}+\frac{\delta}{\sigma_{0}^{(j)}} \Sigma_{1}^{(j)} .
$$

The correction of the centers of phase keeps $\sigma_{0}^{(j)}$ unchanged while setting the dipole moment $\Sigma_{1}^{(j)}$ to zero . Fortunately enough, according to (48), coefficient $S_{0}^{(j)}$ is also zero then. 
Coefficients $u_{2}(x)$ and $u_{2}^{\mathrm{F}}(x)$ still differ by the term $\left(i \kappa \sigma_{0}^{(j)}\right)^{2} u_{0}\left(c_{j}\right)$ but it is easy to restore the agreement at this order too by a slight modification of the Foldy model, actually by substituting the scattering coefficient

$$
w(x)+\frac{i \delta \kappa \sigma_{0}^{(j)}}{1-i \delta \kappa \sigma_{0}^{(j)}} w\left(c_{j}^{\mathrm{corr}}\right) h_{0}^{(1)}\left(\kappa\left|x-c_{j}^{\mathrm{corr}}\right|\right)
$$

for the previous one in (65). It can easily be proved then that the modified Foldy model yields a 2 nd-order approximation in $\delta$ of the actual wave $u_{\delta}$.

Theorem 8 Let the centers of phase and the scattering coefficients be defined according to (79) and (80) respectively. Then the solution $u_{\delta}^{F L}$ corresponding to the Foldy model (67) yields an approximation of the solution to the multi-scattering problem (3) satisfying the following error bound

$$
\left\|u_{\delta}-u_{\delta}^{F L}\right\|_{H^{1}(\mathscr{F} \rho)} \leq C_{\rho} \delta^{3}
$$

under the same conditions and notation already used in Theorem 7.

\section{Extensions and concluding remarks}

A first part of this section is devoted to the consideration of impedance boundary conditions with another asymptotic behavior than the critical one considered above. By considering obstacles that are spheres, we in particular retrieve the wellknown facts that the usual isotropic Foldy model cannot be used to approximate the effect of small sound-hard obstacles. We next give some indications on the adaptations which can be carried out to deal with small penetrable obstacles. To conclude, we give some indications on possible extensions of this study for other asymptotic regimes.

6.1 Multiple scattering problem relative to $N$ spheres

We look at the above multiple scattering problem relative to $N$ spheres of radius $\delta$ but now we consider two additional asymtotic behavior for the surface impedance of the small obstacles. The centers of phase are naturally the centers of the spheres in this configuration. Calculations, too long to be reported here, readily then yield 
the coefficients of the outer expansion of the total wave for all the considered boundary conditions in an explicit way. The 0 -th order coefficient $u_{0}(x)$ is always $u_{\text {inc }}(x)$.

The total wave is now denoted by $u_{\delta, m}(m=0,1,2,3,4)$. The additional subscript $m$ is used to specify to what boundary condition this solution corresponds. In all cases, the total wave has the following asymptotic outer expansion

$$
\begin{gathered}
u_{\delta, m}(x)=u_{\mathrm{inc}}(x)+\delta \sum_{j=1}^{N} h_{0}\left(\kappa\left|x-c_{j}\right|\right) Y_{0,1, m}^{(j)}+\delta^{2} \sum_{j=1}^{N} h_{0}^{(1)}\left(\kappa\left|x-c_{j}\right|\right) Y_{0,2, m}^{(j)}+ \\
\delta^{3} \sum_{j=1}^{N} h_{0}^{(1)}\left(\kappa\left|x-c_{j}\right|\right) Y_{0,3, m}^{(j)}+h_{1}^{(1)}\left(\kappa\left|x-c_{j}\right|\right) Y_{1,3, m}^{(j)} \cdot \frac{x-c_{j}}{\left|x-c_{j}\right|} .
\end{gathered}
$$

Recall that the Hankel function of the first kind can be retrieved from the following expression

$$
h_{n}^{(1)}(z)=z^{n}\left(-z^{-1} \partial_{z}\right)^{n} \frac{e^{i z}}{i z}
$$

(cf., e.g., [25]).

\section{Sound-soft spheres.}

- Boundary condition

$$
u_{\delta, 0}=0 \text { on } \mathscr{S}_{c_{j}}^{\delta}(j=1, \ldots, N),
$$

$\mathscr{S}_{c_{j}}^{\delta}$ being the sphere of center $c_{j}$ and of radius $\delta$.

- 1st-order

$$
Y_{0,1,0}^{(j)}=-i \kappa u_{\text {inc }}\left(c_{j}\right)
$$

- 2nd-order

$$
Y_{0,2,0}^{(j)}=T^{(j)}\left\{Y_{0,1,0}^{(\ell)}\right\}_{\ell=1}^{N}
$$

with

$$
T^{(j)}\left\{v_{\ell}\right\}_{\ell=1}^{N}=-i \kappa\left(v_{j}+\sum_{\ell \neq j} h_{0}^{(1)}\left(\kappa\left|c_{j}-c_{\ell}\right|\right) v_{\ell}\right) .
$$

- 3rd-order

$$
\begin{aligned}
Y_{0,3,0}^{(j)} & =T^{(j)}\left\{Y_{0,2,0}^{(\ell)}\right\}_{\ell=1}^{N}-\frac{i \kappa^{3}}{3} u_{\mathrm{inc}}\left(c_{j}\right) . \\
Y_{1,3,0}^{(j)} & =-i \kappa^{2} \nabla u_{\mathrm{inc}}\left(c_{j}\right)
\end{aligned}
$$

Super-critical acoustic impedance spheres. 
Multiple scattering by small bodies.

45

- Boundary condition

$$
\partial_{\mathbf{n}_{j}} u_{\delta, 1}-\frac{v_{j}}{\delta^{2}} u_{\delta, 1}=0 \text { on } \mathscr{S}_{c_{j}}^{\delta}(j=1, \ldots, N),
$$

- 1st-order

$$
Y_{0,1,1}^{(j)}=-i \kappa u_{\mathrm{inc}}\left(c_{j}\right)
$$

- 2nd-order

$$
Y_{0,2,1}^{(j)}=T^{(j)}\left\{Y_{0,1,1}^{(\ell)}\right\}_{\ell=1}^{N}+\frac{i \kappa}{v_{j}} u_{\mathrm{inc}}\left(c_{j}\right)
$$

- 3rd-order

$$
\begin{aligned}
Y_{0,3,1}^{(j)} & =T^{(j)}\left\{Y_{0,2,1}^{(\ell)}\right\}_{\ell=1}^{N}-\frac{i \kappa^{3}}{3} u_{\text {inc }}\left(c_{j}\right)-\frac{1}{v_{j}} T^{(j)}\left\{Y_{0,1,1}^{(\ell)}\right\}_{\ell=1}^{N}-\frac{i \kappa}{v_{j}^{2}} u_{\text {inc }}\left(c_{j}\right) . \\
Y_{1,3,1}^{(j)} & =-i \kappa^{2} \nabla u_{\text {inc }}\left(c_{j}\right) .
\end{aligned}
$$

Critical acoustic impedance spheres.

- Boundary condition

$$
\partial_{\mathbf{n}_{j}} u_{\delta, 2}-\frac{v_{j}}{\delta} u_{\delta, 2}=0 \operatorname{sur} \mathscr{S}_{c_{j}}^{\delta}(j=1, \ldots, N),
$$

- 1st-order

$$
Y_{0,1,2}^{(j)}=-i \kappa \frac{v_{j}}{1+v_{j}} u_{\mathrm{inc}}\left(c_{j}\right)
$$

- 2nd-order

$$
Y_{0,2,2}^{(j)}=\frac{v_{j}}{1+v_{j}} T^{(j)}\left\{Y_{0,1,2}^{(\ell)}\right\}_{\ell=1}^{N}
$$

- 3rd-order

$$
\begin{aligned}
Y_{0,3,2}^{(j)} & =\frac{v_{j}}{1+v_{j}} T^{(j)}\left\{Y_{0,2,2}^{(\ell)}\right\}_{\ell=1}^{N}-\frac{i \kappa^{3}}{3} u_{\mathrm{inc}}\left(c_{j}\right)+i \kappa^{3} \frac{v_{j}}{\left(1+v_{j}\right)^{2}} u_{\mathrm{inc}}\left(c_{j}\right) \\
Y_{1,3,1}^{(j)} & =-i \kappa^{2} \frac{v_{j}-1}{2+v_{j}} \nabla u_{\mathrm{inc}}\left(c_{j}\right) .
\end{aligned}
$$

Sub-critical acoustic impedance spheres.

- Boundary condition

$$
\partial_{\mathbf{n}_{j}} u_{\delta, 3}-v_{j} u_{\delta, 3}=0 \operatorname{sur} \mathscr{S}_{c_{j}}^{\delta}(j=1, \ldots, N),
$$

- 1st-order

$$
Y_{0,1,3}^{(j)}=0
$$


- 2nd-order

$$
Y_{0,2,3}^{(j)}=-i \kappa v_{j} u_{\mathrm{inc}}\left(c_{j}\right)
$$

- 3rd-order

$$
\begin{aligned}
Y_{0,3,3}^{(j)} & =\left(-\frac{i \kappa^{3}}{3}+i \kappa v_{j}^{2}\right) u_{\mathrm{inc}}\left(c_{j}\right) \\
Y_{1,3,3}^{(j)} & =\frac{i \kappa^{2}}{2} \nabla u_{\mathrm{inc}}\left(c_{j}\right)
\end{aligned}
$$

\section{Sound-hard spheres}

- Boundary condition

$$
\partial_{\mathbf{n}_{j}} u_{\delta, 4}=0 \operatorname{sur} \mathscr{S}_{c_{j}}^{\delta}(j=1, \ldots, N),
$$

- 1st-order

$$
Y_{0,1,4}^{(j)}=0
$$

- 2nd-order

$$
Y_{0,2,4}^{(j)}=0
$$

- 3rd-order

$$
\begin{aligned}
Y_{0,3,4}^{(j)} & =-\frac{i \kappa^{3}}{3} u_{\text {inc }}\left(c_{j}\right) \\
Y_{1,3,4}^{(j)} & =\frac{i \kappa^{2}}{2} \nabla u_{\text {inc }}\left(c_{j}\right) .
\end{aligned}
$$

In view of the above expansions, it can be argued that the usual Foldy model can be retrieved from the critical case just by taking $v_{j}=0$ for high impedance and, as a limiting case, sound-soft spheres. In the opposite case, the above expansions clearly yield that the 1st-order outer expansion reduces to the incident field for weak impedance and sound-hard spheres and can also be derived in a formal way from the critical surface impedance model by letting $v_{j} \rightarrow \infty$. However, the improvement of Foldy's model as done above requires specific expressions for the self-interaction terms.

We also retrieve the well-known fact that dipole effects are involved at the lowest order for hard-sound scatterers. They therefore cannot be handled by means of a simple isotropic usual Foldy's model. 
6.2 Extension to other kinds of scatterers

There is no difficulty to extend the above asymptotic analysis establishing that the scattered field can be approximated at any order of accuracy in $\delta$ by multipoles to the case where the scatterers are characterized by a low impedance boundary condition in the meaning given above for the spheres. However, this extension is not obvious for the other case of a high surface impedance. This is mainly due to the fact that then inner problems are characterized by a boundary condition set in terms of non smooth data

$$
\Pi_{k}^{(j)}=\frac{1}{v_{j}} \partial_{\widehat{\mathbf{n}}_{j}} \Pi_{k-1}^{(j)} \text { on } \partial \widehat{\mathscr{O}^{(j)}} .
$$

Of course, such a difficulty occurs only when the geometry of the scatterer presents some singularity. This probably means that for non smooth scatterers an expansion in integer powers of $\delta$ does not exist. A similar situation is met for other kinds of singular asymptotic expansions (see, e.g., $[10,6])$.

All the above study can be adapted to deal with the case when the small objects are inhomogeneities, in other words, penetrable scatterers. It is enough in this case to substitute the refractive index $n_{j} / \delta^{m}$ to the surface impedance $v_{j} / \delta^{m}$ (see [9]).

\subsection{Concluding remarks}

This study established that the scattered wave by a finite number of small scatterers can be approximated by point scatterers for a fixed frequency at any order of accuracy corresponding to the size of the small obstacles. It has been shown how the usual isotropic Foldy method can be derived in a rigorous way from the firstorder of these approximations for obstacles whose scattering properties are close to that of a sound-soft scatterer. An outcome of the mathematical justification was the possibility to increase the accuracy of this model by suitably locating the center of phase of the monopoles involved in Foldy's model. These results have been obtained for the following asymptotic regime: the frequency, the number of particules, and the distance between any pair of obstacles are assumed to be fixed while the size of the scatterers goes to zero. 
Foldy's model can be used as a part of a homogenization procedure mainly for making up metamaterials (cf., e.g., [27,28,9]). A justification of this approach mainly requires a control on the above bounds relative to the number $N$ of particules and their density. An attempt towards such estimates is given [27,8]. In our opinion however, the issue consisting of defining in a precise way the various asymptotic regimes for such a scattering problem in its full extent largely reamins an open question.

\section{References}

1. Bartoli, N., Bendali, A.: Robust and high-order effective boundary conditions for perfectly conducting scatterers coated by a thin dielectric layer. IMA J. Appl. Math. 67(5), 479-508 (2002)

2. Bendali, A., Cocquet, P.H., Tordeux, S.: Scattering of a scalar time-harmonic wave by $n$ small spheres by the method of matched asymptotic expansions. Numerical Analysis and Applications 5, 116-123 (2012)

3. Bendali, A., Fares, M., Piot, E., Tordeux, S.: Mathematical justification of the Rayleigh cavity model with the method of matched asymptotic expansions. SIAM J. Applied Math. 71(1), 438-459 (2013)

4. Bendali, A., Lemrabet, K.: The effect of a thin coating on the scattering of a time-harmonic wave for the helmholtz equation. SIAM Journal of Applied Mathematics 6(5), 1664-1693 (1996)

5. Bendali, A., Makhlouf, A., Tordeux, S.: Field behavior near the edge of a microstrip antenna by the method of matched asymptotic expansions. Quart. Appl. Math. 69, 691-721 (2011)

6. Caloz, G., Costabel, M., Dauge, M., Vial, G.: Asymptotic expansion of the solution of an interface problem in a polygonal domain with thin layer. Asymptotic Analysis 50(1/2), 121-173 (2006)

7. Cassier, M., Hazard, C.: Multiple scattering of acoustic wave by small sound-soft obstacles in two-dimensions: mathematical justification of the Foldy-Lax model. Wave Motion 50, 18-28 (2013)

8. Challa, D.P., Sini, M.: On the justification of the foldy-lax approximation for the acoustic scattering by small rigid bodies of arbitrary shapes. SIAM Multiscale Model. Simul. 12(1), 58-108 (2014)

9. Cocquet, P.H.: Etude mathématique et numérique de modèles homogénéisés de métamatériaux. Ph.D. thesis, University of Toulouse (2012)

10. Costabel, M., Dauge, M.: A singularly perturbed mixed boundary value problem. Comm. Partial Differential Equations 21(11-12), 16671703 (1996)

11. Cousteix, J., Mauss, J.: Asymptotic Analysis and Boundary Layers. Springer-Verlag, NewYork (2007) 
12. Dyke, M.V.: Perturbation Methods in Fluid Mechanics, annoted edition edn. The Parabolic Press, Stanford, California (1975)

13. Eckhaus, W.: Matched Asymptotic Expansions and Singular Perturbations, North-Holland Mathematics Studies, vol. 6. North-Holland Publishing Company, Amsterdam and London (1973)

14. Foldy, L.: The multiple scattering of waves. I. General theory of isotropic scattering by randomly distributed scatterers. Phys. Rev. 67, 107-119 (1945)

15. Gumerov, N.A., Duraiswamy, R.: Fast Multipole Method for the Helmholtz Equation in Three Dimensions. Elsevier, Amsterdam (2004)

16. Hsiao, G.C., Wendland, W.L.: Boundary Iintegral Equations. Springer, Berlin-Heidelberg (2008)

17. Huang, K., Li, P.: A two-scale multiple scattering problem. SIAM Multiscale Model. Simul. 8(4), 1511-1534 (2010)

18. Jacobsen, F., Juhl, P.M.: Fundamentals of General Linear Acoustics. Wiley and Sons Ltd, Chichester, West Sussex, United Kingdom (2013)

19. Joly, P., Tordeux, S.: Matching of Asymptotic Expansions for Wave Propagation in Media with Thin Slots i: The Asymptotic Expansion. Multiscale Modeling and Simulation: A SIAM Interdisciplinary Journal 5(1), 304-336 (2006)

20. Laurens, S., Tordeux, S., Bendali, A., Fares, M., Kotiuga, P.R.: Lower and upper bounds for the rayleigh conductivity of a perforated plate. ESAIM: Mathematical Modelling and Numerical Analysis 47(4), 675-696 (2013)

21. Liao, J., Ji, C.: Extended FoldyLax approximation on multiple scattering. Mathematical Modelling and Analysis 10(1), 85-98 (2014)

22. Martin, P.A.: Multiple Scattering Intercation of Time-Harmonic Waves with N Obstacles. Cambridge University Press, Cambridge (2006)

23. Maz'ya, V.G., Poborchi, S.V.: Extension of functions in sobolev spaces on parameter dependent domains,. Mathematische Nachrichten 178(1), 5-41 (1996)

24. Nédélec, J.C.: Acoustic and Electromagnetic Equations: Integral Representations for Harmonic Problems. Springer, Berlin (2001)

25. Olver, F.W., Lozier, D.W., Boisvert, R.F., Clark, C.W.: NIST Handbook of Mathematical Functions. NIST U.S. Department of Commerce and Cambridge University Press, NewYork (2010)

26. Ramm, A.G.: Wave scattering by small bodies of arbitrary shapes. World Scientific Publishing Co. Inc. (2005)

27. Ramm, A.G.: Many-body wave scattering by small bodies and applications. Journal of Mathematical Physics 48, 103,511-1-103,511-28 (2007)

28. Ramm, A.G.: Wave scattering by small bodies and creating materials with a desired refraction coefficient. Afr. Mat. 22, 33-55 (2011)

29. Taylor, M.: Partial Differential Equations I, Basic Theory. Springer-Verlag, New York (1996) 
30. Wang, Z.X., Guo, D.R.: Special Functions. World Scientific Publishing Co. Inc., Teaneck, New-Jersey (1989)

31. Wilcox, C.H.: Scattering theory for the d'Alembert equation in exterior domains, vol. 442. Springer-Verlag, Berlin (1975)

32. Zhong, Y., Chen, X.: Music imaging and electromagnetic inverse scattering of multiplescattering small anisotropic spheres. IEEE Transactions on Antennas and Propagation 55(12), 3542-3549 (2007) 